\title{
Master functions and equations for perturbations of vacuum spherically symmetric spacetimes
}

\author{
Michele Lenzi® $\oplus^{1,2,3}$ and Carlos F. Sopuerta $\circledast^{1,2}$ \\ ${ }^{1}$ Institut de Ciències de l'Espai (ICE, CSIC), Campus UAB, Carrer de Can Magrans s/n, \\ 08193 Cerdanyola del Vallès, Spain \\ ${ }^{2}$ Institut d'Estudis Espacials de Catalunya (IEEC), Edifici Nexus, Carrer del Gran Capità 2-4, \\ despatx 201, 08034 Barcelona, Spain \\ ${ }^{3}$ Dipartimento di Fisica e Astronomia, Università di Bologna, via Irnerio 46, 40126 Bologna, Italy
}

(Received 23 August 2021; accepted 8 September 2021; published 14 October 2021)

\begin{abstract}
Perturbation theory of vacuum spherically symmetric spacetimes is a crucial tool for understanding the dynamics of black hole perturbations. Spherical symmetry allows for an expansion of the perturbations in scalar, vector, and tensor harmonics. The resulting perturbative equations are decoupled for modes with different parity and different harmonic numbers. Moreover, for each harmonic and parity, the equations for the perturbations can be decoupled in terms of (gauge-invariant) master functions that satisfy $1+1$ wave equations. By working in a completely general perturbative gauge, in this paper we study what is the most general master function that is linear in the metric perturbations and their first-order derivatives and satisfies a wave equation with a potential. The outcome of the study is that for each parity we have two branches of solutions with similar features. One of the branches includes the known results: In the odd-parity case, the most general master function is an arbitrary linear combination of the Regge-Wheeler and the Cunningham-Price-Moncrief master functions whereas in the even-parity case it is an arbitrary linear combination of the Zerilli master function and another master function that is new to our knowledge. The other branch is very different since it includes an infinite collection of potentials which in turn lead to an independent collection master of functions which depend on the potential. The allowed potentials satisfy a nonlinear ordinary differential equation. Finally, all the allowed master functions are gauge invariant and can be written in a fully covariant form.
\end{abstract}

DOI: 10.1103/PhysRevD.104.084053

\section{INTRODUCTION}

Spacetime perturbation theory is one of the main tools in general relativity to describe physical systems and make reliable predictions about their characteristics and dynamical behavior. It has been applied essentially to all the main problems in the area of relativistic astrophysics and cosmology: from the origin and growth of cosmological structures and the cosmic microwave background [1-4] to phenomena involving relativistic stars and black holes [58], including gravitational wave generation and propagation [9-12] (see [12-18] for applications of black hole perturbation theory to gravitational wave astronomy). There are also many applications of black hole perturbation theory that have implications for fundamental physics (see [19-21] for reviews).

The long experience in relativistic perturbation theory tells us that it is a very powerful tool, in part because starting from very simplified situations, encoded in what is known as the background spacetime, it has shown to provide results and predictions that many times go beyond the range of applicability that one may expect from backof-the-envelope estimates. For example, this has happened when we have compared the outcome of full numerical relativity simulations with post-Newtonian/Minkowskian theories and relativistic perturbation theory.

In this work we focus on relativistic perturbation theory of vacuum spherically symmetric spacetimes. We allow for the presence of a cosmological constant which, as we are going to see, does not make our computations much more complicated since it can be incorporated into our equations in a relatively simple way. This means that our study includes the dynamics of perturbations around Schwarzschild, Schwarzschild-de Sitter, and Schwarzschild-anti-de Sitter spacetimes, including also the associated maximally symmetric spacetimes: Minkowski, de Sitter, and anti-de Sitter respectively. It is well known that perturbations of spherically symmetric spacetimes can be decomposed in spherical harmonics, in such a way that the associated equations for the different harmonic modes decouple. Moreover, the equations for different parity harmonics also decouple, i.e., odd-parity (axial) modes can be treated separately from even-parity (polar) modes. Another crucial feature of the theory of perturbations of spherically symmetric spacetimes is that, 
for each harmonic and parity, it is possible to construct master functions, made out of linear combinations of the metric perturbations and their first-order derivatives, that satisfy $1+1$ wave (master) equations decoupled from the rest of metric perturbations. The characteristic curves of the wave operator of the master equations are fully determined by the background (Lorentzian) metric. In some perturbative gauges, it is even possible to reconstruct all the metric perturbations in terms of the solutions of the master equations, something that is required in some problems, like in the self-force program [22-24].

In the case of perturbations of the Schwarzschild metric, the first steps [25] were already taken in the 1950s by Regge and Wheeler [26] (see also [27,28]), who managed to decouple the equations for odd-parity perturbations in the gauge named after them (also the master function and equation are named after them). However, it took a while until the same was done for even-parity perturbations, when Zerilli found the way to decouple the perturbative equations [29,30] (see also [31]). Starting from these pioneering works, there have been many interesting developments in perturbation theory of spherically symmetric spacetimes: gauge-invariant and/or explicitly covariant formalisms [3235] (see [36,37] for the case of D-dimensional maximally symmetric spacetimes), quasinormal modes [5,6,38-40] (for $D$ dimensions see [41]), stability of dynamics of the perturbations [42,43], stability of black holes in de Sitter Space [44], etc. For reviews on nonrotating black hole perturbation theory see [34,45-48] (for second-order perturbations see [49-51]). On the other hand, similar developments took place for perturbations of rotating black holes $[52,53]$. For studies of the stability of black holes in asymptotically flat spacetimes see $[54,55]$.

In this paper, we further investigate the construction of master functions and equations. In particular, we focus on the following questions: What is the most general master function that decouples the equations for the metric perturbations of spherically symmetric vacuum spacetimes? And, what are the possible potentials associated with those master functions? To answer these questions we assume that the master functions are linear combinations (with coefficients that depend only on the radial areal coordinate, $r$ ) of the metric perturbations and their firstorder derivatives. Our analysis turns out to be very similar for the odd- and even-parity cases, also leading us to similar conclusions. The result we find is that we can distinguish two branches of solutions (for each parity and harmonic mode). The first branch is mostly known: The master functions are linear combinations of two different (linearly independent) master functions. In the odd-parity case they can be taken to be the Regge-Wheeler [26] and the Cunningham-Price-Moncrief [56-58] master functions. In the even-parity case, one of them can be taken to be the well-known Zerilli-Moncrief master function [29-31], while the second one, as far as we know, was previously unknown. It turns out that, for both parities, the independent master functions can be chosen so that one of them is the time derivative of the other one. Regarding the master equations themselves, which in our case are essentially determined by the potential, in the first branch we find the well-known potentials: the Regge-Wheeler potential for odd-parity perturbations and the Zerilli potential for evenparity perturbations. On the other side, the second branch was essentially unknown. To begin with, there are infinite possible potentials, different from the ones already known in the first branch. Actually, the allowed potentials satisfy a nonlinear ordinary differential equation. The master functions are again a linear combination (again with coefficients that depend only on $r$ ) of two independent master functions. In the odd-parity case, they can be taken to be the Cunningham-Price-Moncrief master function and a new one that combines the Cunningham-PriceMoncrief and another gauge-invariant function. The even-parity case is analogous, the most general master function is a linear combination of the Zerilli-Moncrief master function and another new master function that contains the Zerilli-Moncrief master function and a gaugeinvariant variable.

Some remarkable features and consequences coming from this study are the following: (i) All the master functions involved are automatically gauge invariant. (ii) All the master functions and master equations admit a fully covariant form with respect to the $1+1$ Lorentzian metric. (iii) In the same way that the Regge-Wheeler and Zerilli potentials coincide for the case of a maximally symmetric background, the equations for the potentials in the second branch also coincide. (iv) Our approach constitutes an original and systematic way of searching for master functions and equations without having to resort to look for ad hoc combinations of the perturbative field equations that yield decoupled master equations.

This paper is organized as follows: In Sec. II we introduce all the necessary elements of relativistic perturbation theory for (vacuum) spherically symmetric spacetimes, including the elements associated with the background spacetime, the decomposition of the perturbations in spherical harmonics, gauge invariance, and the known master functions in different forms. In Sec. III, we describe how to obtain the most general master functions and equations satisfying the hypothesis mentioned above. Finally, in Sec. IV we summarize and discuss the results of this paper. We also include two appendixes with key formulas used in this work. We use geometric units in which $G=c=1$.

\section{RELATIVISTIC PERTURBATION THEORY OF SPHERICALLY SYMMETRIC SPACETIMES}

In this section we introduce all the ingredients and machinery needed to derive the main results of this work. 


\section{A. Basics of relativistic perturbation theory}

Relativistic perturbation theory is usually formulated by assuming the existence of a one-parameter family of spacetimes, $\left(\mathcal{M}_{\lambda}, g_{\lambda}\right)$, in such a way that the perturbations are constructed as a Taylor expansion of this family around the $\lambda=0$ spacetime (see, e.g., $[59,60]$ ), the background spacetime, which usually represents an idealized gravitational system, typically with a high degree of symmetry as in our case. Here, we assume the background ${ }^{1}$ to be a vacuum (including the cosmological constant) spherically symmetric spacetime. Then, the background spacetime metric, $\hat{g}_{\mu \nu}$, satisfies the vacuum Einstein's field equations including the cosmological constant term:

$$
\hat{G}_{\mu \nu}=\hat{R}_{\mu \nu}-\frac{1}{2} \hat{g}_{\mu \nu} \hat{R}+\Lambda \hat{g}_{\mu \nu}=0,
$$

where $\hat{R}_{\mu \nu}$ and $\hat{G}_{\mu \nu}$ denote the Ricci and Einstein tensors of the background metric respectively, $\hat{R}=\hat{g}^{\mu \nu} \hat{R}_{\mu \nu}$ is the background scalar curvature, and $\Lambda$ is the cosmological constant.

In this framework, the perturbations are defined as the derivative terms of the Taylor series expansion, evaluated on the background. The parameter $\lambda$ controls the strength of the perturbations and in most applications it is a formal parameter without a specific physical meaning, except in some cases in which it is identified with some relevant physical parameter of the system (see, e.g., $[59,61,62]$ for more details on the formulation of relativistic perturbation theory). Since in our case $\lambda$ is a formal parameter, we are going to ignore it from now on for the sake of simplicity. The physical (perturbed) spacetime, a member of the oneparameter family of spacetimes $\left(\mathcal{M}_{\lambda}, g_{\lambda}\right)$, is endowed with a metric $g_{\mu \nu}$ which, once a correspondence between the background spacetime is established, can be constructed to linear order from the background solution $\hat{g}_{\mu \nu}$ and the metric perturbations $h_{\mu \nu}\left(\left|h_{\mu \nu}\right| \ll\left|\hat{g}_{\mu \nu}\right|\right)$ via the relation

$$
g_{\mu \nu}=\hat{g}_{\mu \nu}+h_{\mu \nu}
$$

For any quantity $Q$, we denote the deviations between the perturbative and background expressions with a $\delta$ in front of the original quantity, i.e., $\delta Q=Q-\hat{Q}$, where $Q$ is the expression from the perturbed/physical spacetime. In this way, $h_{\mu \nu}=\delta g_{\mu \nu}=g_{\mu \nu}-\hat{g}_{\mu \nu}$. When we expand such a quantity in the different perturbative orders we are actually performing Taylor expansions in the parameter $\lambda$. In this sense, at first order, the perturbed Christoffel symbols can be written in terms of the metric perturbations $h_{\mu \nu}$ and their covariant derivatives with respect to the background metric (denoted here by a semicolon), as follows:

\footnotetext{
${ }^{1}$ We use a hat to denote quantities associated with the background spacetime, like $\hat{Q}$.
}

$$
\delta \Gamma_{\mu \nu}^{\rho}=\frac{1}{2} \hat{g}^{\rho \sigma}\left(h_{\mu \sigma ; \nu}+h_{\nu \sigma ; \mu}-h_{\mu \nu ; \sigma}\right) .
$$

From the expression of these quantities we deduce that they are tensors with respect to coordinate changes in the background spacetime. Then, we can write the perturbations of the Riemann tensor in terms of the perturbed Christoffel symbols (which are tensors from the point of view of the background spacetime) as follows:

$$
\delta R^{\mu}{ }_{\nu \rho \sigma}=\delta \Gamma_{\nu \sigma ; \rho}^{\mu}-\delta \Gamma_{\nu \rho ; \sigma}^{\mu}=2 \delta \Gamma_{\nu[\sigma ; \rho]}^{\mu} .
$$

In the same way, the perturbations of the Ricci tensor can be written in terms of the covariant derivatives of the perturbed Christoffel symbols:

$$
\delta R_{\mu \nu}=\delta \Gamma_{\mu \nu ; \rho}^{\rho}-\delta \Gamma_{\rho \mu ; \nu}^{\rho} .
$$

The Einstein tensor can be decomposed as $G_{\mu \nu}=\hat{G}_{\mu \nu}+$ $\delta G_{\mu \nu}=\delta G_{\mu \nu}$, where the second equality holds by virtue of the Einstein field equations satisfied by the background metric [Eq. (1)]. The perturbation in the Einstein tensor in terms of the metric perturbations $h_{\mu \nu}$ are

$\delta G_{\mu \nu}=-\frac{1}{2} \hat{\square} \bar{h}_{\mu \nu}-\hat{R}_{\mu}^{\rho}{ }_{\mu \nu}{ }_{\nu} \bar{h}_{\rho \sigma}+\hat{\nabla}_{(\mu} \mathcal{L}_{\nu)}-\frac{1}{2} \hat{g}_{\mu \nu}\left(\hat{\nabla}_{\rho} \mathcal{L}^{\rho}\right)$,

where $\hat{R}^{\rho}{ }_{\mu \nu}{ }_{\nu}$ is the background Riemann tensor and we have used again the background Einstein's field equations [Eq. (1)]: $\hat{G}_{\mu \nu}=0 \Rightarrow \hat{R}_{\mu \nu}=\Lambda \hat{g}_{\mu \nu}$ and $\hat{R}=\hat{g}^{\mu \nu} \hat{R}_{\mu \nu}=4 \Lambda$. Moreover, we have introduced several definitions in Eq. (6). First, we have introduced the trace-reversed metric perturbations:

$$
\bar{h}_{\mu \nu}=h_{\mu \nu}-\frac{1}{2} \hat{g}_{\mu \nu} h,
$$

with $h$ being the trace of $h_{\mu \nu}$ with respect to the background metric

$$
h=\hat{g}^{\mu \nu} h_{\mu \nu}
$$

Second, we have introduced the d'Alambertian associated with the background:

$$
\hat{\square} \bar{h}_{\mu \nu}=\bar{h}_{\mu \nu ; \rho} ; \rho, \quad \hat{\square} h=h_{; \rho} ; \rho .
$$

And finally, we have introduced the quantity

$$
\mathcal{L}_{\mu}=\hat{g}^{\rho \sigma} \hat{\nabla}_{\rho} \bar{h}_{\sigma \mu}
$$

When we impose $\mathcal{L}_{\mu}=0$ we are in the so-called Lorenz gauge. But in this paper we are not going to impose any particular gauge, i.e., the developments we present are completely general. 


\section{B. Background solution: Vacuum spherically symmetric spacetimes}

We consider background spacetimes, or regions of spacetimes, that are solutions of the Einstein vacuum equations including a cosmological constant [see Eq. (1)]. These solutions come from a generalization of Birkhoff's local uniqueness theorem [63] (published before by Jebsen $[64,65]$; see also $[66,67]$ ) to the case of a nonvanishing cosmological constant [68] (see [69] for details). It turns out that the only locally spherically symmetric solutions to Eqs. (1) (see [69]) are locally isometric either to one of the Schwarzschild-de Sitter (SchdS [70]) and Schwarzschildanti-de Sitter (SchAdS) solutions or to the Nariai spacetime [71,72], which can be seen as the limit of SchdS when the cosmological and event horizons coincide ${ }^{2}$ (see [73]). This family of metrics includes very important solutions as the maximally symmetric solutions of Einstein equations: Minkowski flat spacetime (M; $\Lambda=0)$, de $\operatorname{Sitter}(\mathrm{dS} ; \Lambda>0)$, and anti-de Sitter (AdS; $\Lambda<0$ ). Locally, the background metric can be written in the so-called Schwarzschild form,

$$
d s^{2}=\hat{g}_{\mu \nu} d x^{\mu} d x^{\nu}=-f(r) d t^{2}+\frac{d r^{2}}{f(r)}+r^{2} d \Omega^{2},
$$

where $d \Omega^{2}=d \theta^{2}+\sin ^{2} \theta d \varphi^{2}$ is the line element of the 2 -sphere and $f(r)$ is a function parametrizing time translations (related to the redshift).

The solutions described by the metric (11) satisfy Einstein's equations (1), which become ordinary differential equations (ODEs) for $f(r)$ :

$$
\begin{aligned}
& r f^{\prime}+f+\Lambda r^{2}-1=0, \\
& r\left(f^{\prime \prime}+2 \Lambda\right)+2 f^{\prime}=0 .
\end{aligned}
$$

There are two combinations of $f(r)$ and its derivatives that are constants and correspond to the cosmological constant $\Lambda$ and the spacetime mass $M$ respectively:

$$
\begin{gathered}
\Lambda=-\frac{1}{2 r^{2}}\left(r^{2} f^{\prime}\right)^{\prime}, \\
M=\frac{r}{2}\left(1-f-\frac{\Lambda}{3} r^{2}\right)=\frac{r}{2}\left[1-f+\frac{1}{6}\left(r^{2} f^{\prime}\right)^{\prime}\right] .
\end{gathered}
$$

In the case of Schwarzschild spacetime [25] (also found independently by Droste [74]) we have

$$
f_{\mathrm{Sch}}=1-\frac{r_{s}}{r}
$$

\footnotetext{
${ }^{2}$ We will not consider here the particular case of the Nariai metric as it may require a particular treatment.
}

where $r_{s}$ is the Schwarzschild radius, $r_{s}=2 G M / c^{2}=2 M$. In the case of de Sitter and anti-de Sitter spacetimes we have

$$
f_{\mathrm{dS}}=1-\frac{r^{2}}{L^{2}}, \quad f_{\mathrm{AdS}}=1+\frac{r^{2}}{L^{2}}
$$

where $L$ is the (anti-)de Sitter length scale, which determines the cosmological constant as follows:

$$
\Lambda= \pm \frac{3}{L^{2}}
$$

where the plus sign corresponds to de Sitter and the minus sign to anti-de Sitter. Apart from these two cases, we have the case of the Schwarzschild-de Sitter (SchdS) spacetime, which contains the previous two cases in the limits $M \rightarrow 0$ (dS) and $L \rightarrow \infty$ (Sch). This last limit is equivalent to $\Lambda \rightarrow 0$. The function $f(r)$ for Schwarzschild-de Sitter and Schwarzschild-anti-de Sitter is

$$
f_{\text {SchdS } / \text { SchAdS }}(r)=1-\frac{2 M}{r}-\frac{\Lambda}{3} r^{2},
$$

where $\Lambda$ is given in Eq. (18).

\section{Multipolar expansion of the perturbations of vacuum spherically symmetric spacetimes}

The background metric can be written as the warped product of two manifolds: $M^{2} \times{ }_{r} S^{2}$, where $M^{2}$ is a twodimensional Lorentzian manifold, $r$ is the radial area coordinate, and $S^{2}$ denotes the 2 -sphere. Therefore, the background metric is given by the semidirect product of a Lorentzian metric on $M^{2}, g_{a b}$, and the unit curvature metric on $S^{2}, \Omega_{A B}$ :

$$
\hat{g}_{\mu \nu}=\left(\begin{array}{cc}
g_{a b} & 0 \\
0 & r^{2} \Omega_{A B}
\end{array}\right) .
$$

Coordinates on $M^{2}$ are going to be denoted with lowercase Latin indices, $\left(x^{a}\right)=(t, r)$. Coordinates on $S^{2}$ are denoted with uppercase Latin indices as $\left(\Theta^{A}\right)=(\theta, \varphi)$. Then, in connection with Eq. (11) we can write

$$
\begin{gathered}
g_{a b} d x^{a} d x^{b}=-f(r) d t^{2}+\frac{d r^{2}}{f(r)}, \\
\Omega_{A B} d \Theta^{A} d \Theta^{B}=d \theta^{2}+\sin ^{2} \theta d \varphi^{2} .
\end{gathered}
$$

We use a vertical bar to denotes covariant differentiation on the two-sphere $S^{2}$ (then $\Omega_{A B \mid C}=0$ ). Similarly, we use a colon to denote covariant differentiation with respect to the metric of the Lorentzian two-dimensional manifold $M^{2}$, i.e., $g_{a b: c}=0$. On the other hand, the antisymmetric covariant unit tensor associated with the volume form 
(Levi-Civita tensor) in $S^{2}$ is denoted by $\epsilon_{A B}$, and the corresponding one on the Lorentzian manifold $M^{2}$ is denoted by $\varepsilon_{a b}$.

The particular geometric structure of the background implies that for certain quantities (e.g., solutions of the wave equation in the background) we can separate the dependence on the coordinates of $M^{2}$ from the angular dependence, which, in turn, can be expanded in spherical harmonics. The different harmonics can be divided into even- and odd-parity harmonics depending on how they transform under a parity transformation, $(\theta, \phi) \rightarrow(\pi-\theta, \phi+\pi)$. If a given harmonic object $\mathcal{O}^{\ell m}$ transforms as $\mathcal{O}^{\ell m} \rightarrow(-1)^{\ell} \mathcal{O}^{\ell m}$ it is said to be of the even-parity type; while if it transforms as $\mathcal{O}^{\ell m} \rightarrow(-1)^{\ell+1} \mathcal{O}^{\ell m}$ it is said to be of the odd-parity type. With this in mind, the scalar, vector, and tensor spherical harmonics are as follows:

(i) The scalar harmonics $Y^{\ell m}$ are eigenfunctions of the Laplace operator on the two sphere (see Appendix A):

$$
\Omega^{A B} Y_{\mid A B}^{\ell m}=-\ell(\ell+1) Y^{\ell m} .
$$

(ii) The vector spherical harmonics, which are defined for $\ell \geq 1$, are given by

$$
\begin{gathered}
Y_{A}^{\ell m} \equiv Y_{\mid A}^{\ell m} \quad \text { even (polar) parity, } \\
X_{A}^{\ell m} \equiv-\epsilon_{A}^{B} Y_{B}^{\ell m} \quad \text { odd (axial) parity. }
\end{gathered}
$$

(iii) The basis of symmetric second-rank tensor spherical harmonics, which are defined for $\ell \geq 2$, are given by

$$
\begin{gathered}
T_{A B}^{\ell m} \equiv Y^{\ell m} \Omega_{A B} \quad \text { even parity, } \\
Y_{A B}^{\ell m} \equiv Y_{\mid A B}^{\ell m}+\frac{\ell(\ell+1)}{2} Y^{\ell m} \Omega_{A B} \quad \text { even parity, } \\
X_{A B}^{\ell m} \equiv X_{(A \mid B)}^{\ell m} \quad \text { odd parity. }
\end{gathered}
$$

Differential properties of these spherical harmonics that are necessary to manipulate the perturbative Einstein equations are given in Appendix A.

The metric perturbations can be written as a multipole expansion using scalar $\left(Y^{\ell m}\right)$, vector $\left(Y_{A}^{\ell m}, X_{A}^{\ell m}\right)$, and tensor spherical harmonics $\left(T_{A B}^{\ell m}, Y_{A B}^{\ell m}, X_{A B}^{\ell m}\right)$. The main reason for expanding the metric perturbations in this way is that the underlying spherical symmetry prevents different harmonics and different parity modes from mixing, and the perturbation equations can be obtained for each $(\ell, m)$ and parity mode separately (see, e.g., $[32,33]$ ):

$$
h_{\mu \nu}=\sum_{\ell, m} h_{\mu \nu}^{\ell m, \text { odd }}+h_{\mu \nu}^{\ell m, \text { even }}
$$

where

$$
h_{\mu \nu}^{\ell m, \text { odd }}=\left(\begin{array}{cc}
0 & h_{a}^{\ell m} X_{A}^{\ell m} \\
* & h_{2}^{\ell m} X_{A B}^{\ell m}
\end{array}\right),
$$

and

$h_{\mu \nu}^{\ell m, \text { even }}=\left(\begin{array}{cc}h_{a b}^{\ell m} Y^{\ell m} & \mathrm{~J}_{a}^{\ell m} Y_{A}^{\ell m} \\ * & r^{2}\left(K^{\ell m} T_{A B}^{\ell m}+G^{\ell m} Y_{A B}^{\ell m}\right)\end{array}\right)$.

Here the asterisk denotes the symmetry on the tensor components, $K^{\ell m}$ and $S^{\ell m}$ denote the scalar perturbations, $h_{2}^{\ell m}$ and $h_{A}^{\ell m}$ the vector perturbations, and $h_{a b}^{\ell m}$ the tensorial ones. All of them depend only on the coordinates $\left\{x^{a}\right\}$ of $M^{2}$.

\section{Gauge invariance}

In relativistic perturbation theory $[59,61]$ there is a gauge freedom associated with the infinite possible ways of choosing the correspondence between the background and physical spacetimes (see Sec. II A). In practical terms, this freedom can be associated with the different ways in which we can identify points of the two spacetimes. Taking into account that we can pull back the physical metric into the background tensorial structure [as described by Eq. (2)], different choices of correspondence between the background and physical spacetimes can be used (from the point of view of the background spacetime) and a coordinate change of the type

$$
x^{\mu} \rightarrow x^{\prime \mu}=x^{\mu}+\xi^{\mu},
$$

where $x^{\mu}$ and $x^{\prime \mu}$ are the coordinates of two points of the physical spacetime, say $p$ and $p^{\prime}$, that have been identified with a single point of the background spacetime, say $\bar{p}$, by two mappings between the two spacetimes. The mapping between the two points $p$ and $p^{\prime}$ constitutes what we call a gauge transformation in perturbation theory, and Eq. (32) is the coordinate version of such a gauge transformation. The difference between the coordinates of the two points $p$ and $p^{\prime}$ (as seen from the background spacetime) is described by a vector field, $\xi^{\mu}$, which is the local generator of the gauge transformation, and which is assumed to be small in the same way as we assume that the perturbations are small $\left(\left|\xi^{\mu}\right| \ll\left|\hat{g}_{\mu \nu}\right|\right)$.

The gauge transformation in Eq. (32) generates the following transformation of the metric perturbations:

$$
h_{\mu \nu} \rightarrow h_{\mu \nu}^{\prime}=h_{\mu \nu}-2 \xi_{(\mu ; \nu)} .
$$

It is important to understand how a general gauge transformation changes the harmonic components of the metric perturbations. To that end, in the same way we have decomposed the metric perturbations in spherical harmonics we have to do the same with the generator of the gauge transformation $\xi^{\mu}$. For even-parity perturbations, the $(\ell, m)$ 
harmonic of the gauge generator can be written in the form

$$
\xi_{\mu}^{\ell m, \text { even }} d x^{\mu}=\alpha_{a}^{\ell m}\left(x^{b}\right) d x^{a}+r^{2} \beta^{\ell m}\left(x^{a}\right) Y_{A}^{\ell m} d \Theta^{A},
$$

and for odd-parity perturbations

$$
\xi_{\mu}^{\ell m, \text { odd }} d x^{\mu}=r^{2} \gamma^{\ell m}\left(x^{a}\right) X_{A}^{\ell m} d \Theta^{A} .
$$

Note that there are three gauge functions for even-parity perturbation and just one for the odd-parity ones.

Introducing the multipolar decomposition of the metric perturbations and the gauge vector into Eq. (33), we find that the even-parity metric perturbations transform as follows:

$$
\begin{gathered}
h_{a b}^{\ell \ell m}=h_{a b}^{\ell m}-2 \alpha_{(a: b)}^{\ell m}, \\
\mathbf{J}_{a b}^{\ell \ell m}=\mathbf{J}_{a}^{\ell m}-\left(\alpha_{a}^{\ell m}+r^{2} \beta_{: a}^{\ell m}\right), \\
K^{\prime \ell m}=K^{\ell m}+\ell(\ell+1) \beta^{\ell m}-2 \frac{r^{: a}}{r} \alpha^{\ell m}, \\
G^{\ell \ell m}=G^{\ell m}-2 \beta^{\ell m} .
\end{gathered}
$$

And the odd-parity metric perturbations transform according to

$$
\begin{aligned}
& h_{a}^{\prime \ell m}=h_{a}^{\ell m}-r^{2} \gamma_{: a}^{\ell m}, \\
& h_{2}^{\prime \ell m}=h_{2}^{\ell m}-2 r^{2} \gamma^{\ell m} .
\end{aligned}
$$

There are combinations of the metric perturbations and its derivatives that are invariant under gauge transformations. In the case of even-parity metric perturbations there are four independent gauge-invariant quantities, which can be written as

$$
\begin{gathered}
\tilde{h}_{a b}=h_{a b}-\kappa_{a: b}-\kappa_{b: a}, \\
\tilde{K}=K+\frac{\ell(\ell+1)}{2} G-2 \frac{r^{a}}{r} \kappa_{a},
\end{gathered}
$$

where

$$
\kappa_{a}=\mathrm{J}_{a}-\frac{r^{2}}{2} G_{: a}, \quad r_{a}=r_{: a} \Rightarrow r^{a}=g^{a b} r_{b} .
$$

In the case of odd-parity metric perturbations there are two independent gauge-invariant quantities:

$$
\tilde{h}_{a}=h_{a}-\frac{1}{2} h_{2: a}+\frac{r_{a}}{r} h_{2}
$$

\section{E. Known master functions and equations}

Before entering in the search for master functions and equations, let us review the most important known master functions and how they are expressed in covariant form (with respect to the metric $g_{a b}$ of $M^{2}$; see Sec. II C). For odd-parity perturbations, the first master function was introduced by Regge and Wheeler [26] in a pioneering work on black hole perturbation theory. The covariant form of this master function is (see, e.g., [48])

$$
\Psi_{\mathrm{RW}}=\frac{r^{a}}{r} \tilde{h}_{a}
$$

One can alternatively use the master function introduced by Cunningham, Price, and Moncrief [56-58], which in covariant form reads (see also $[48,75]$ )

$$
\Psi_{\mathrm{CPM}}=\frac{2 r}{(\ell-1)(\ell+2)} \varepsilon^{a b}\left(\tilde{h}_{b: a}-\frac{2}{r} r_{a} \tilde{h}_{b}\right) .
$$

A classification of odd-parity master functions can be found in [47].

In the case of even-parity perturbations we have the master function introduced by Zerilli [30] and later by Moncrief [31] (see also [48,76]). It admits the following covariant expression:

$$
\Psi_{\mathrm{ZM}}=\frac{2 r}{\ell(\ell+1)}\left\{\tilde{K}+\frac{2}{\lambda}\left(r^{a} r^{b} \tilde{h}_{a b}-r r^{a} \tilde{K}_{: a}\right)\right\},
$$

where

$$
\begin{aligned}
\lambda(r) & =r f^{\prime}-2(f-1)+(\ell+2)(\ell-1) \\
& =(\ell+2)(\ell-1)-\Lambda r^{2}-3(f-1),
\end{aligned}
$$

which in the Schwarzschild case reduces to

$$
\lambda(r)=(\ell-1)(\ell+2)+\frac{3 r_{s}}{r} .
$$

All these master functions satisfy wave-type equations in $1+1$ dimensions, with respect to the metric $g_{a b}$ of the Lorentzian manifold $M^{2}$, with a potential term. The form of these equations in the case of vacuum perturbation looks as follows:

$$
\left(\square_{2}-\Omega_{\text {even/odd }}\right) \Psi_{\text {even/odd }}=0,
$$

where $\Psi_{\text {even/odd }}(t, r)$ is the even/odd master function of choice; $\Omega_{\text {even/odd }}(r)$ is the potential, which only depends on the radial area coordinate $r$; and the action of the operator $\square_{2}$ on any scalar field $\phi$ is given by

$$
\square_{2} \phi=g^{a b} \phi_{: a b} .
$$


A slightly different way of introducing the potential comes from the expression of the operator $\square_{2}$ in Schwarzschild coordinates

$$
\square_{2} \phi=-\frac{1}{f} \frac{\partial^{2} \phi}{\partial t^{2}}+\frac{\partial}{\partial r}\left(f \frac{\partial \phi}{\partial r}\right) .
$$

At this point we can introduce the tortoise coordinate:

$$
\frac{d r_{*}(r)}{d r}=\frac{1}{f(r)} .
$$

Combining this with Eq. (53) we can write the master equation (51) in the following more familiar form:

$$
\left(-\frac{\partial^{2}}{\partial t^{2}}+\frac{\partial^{2}}{\partial r_{*}^{2}}-V_{\text {even } / \text { odd }}\right) \Psi_{\text {even } / \text { odd }}=0,
$$

where the potential $V_{\text {even/odd }}$, is related to the one in Eq. (51) by

$$
V_{\text {even } / \text { odd }}=f \Omega_{\text {even } / \text { odd }} .
$$

In most places the potential that is used is $V_{\text {even/odd }}$, but in this work we will use both.

To finish this section, we just recall that in the case of the Schwarzschild spacetime, the Regge-Wheeler potential is given by

$$
\Omega_{\text {odd }}(r)=\frac{\ell(\ell+1)}{r^{2}}-\frac{3 r_{s}}{r^{3}},
$$

while the Zerilli potential is

$$
\begin{aligned}
\Omega_{\mathrm{even}}(r)= & \frac{1}{\lambda^{2}}\left[\frac{(\ell-1)^{2}(\ell+2)^{2}}{r^{2}}\left(\ell(\ell+1)+\frac{3 r_{s}}{r}\right)\right. \\
& \left.+\frac{9 r_{s}^{2}}{r^{4}}\left((\ell-1)(\ell+2)+\frac{r_{s}}{r}\right)\right],
\end{aligned}
$$

where $\lambda(r)$ is defined in Eq. (49), and for Schwarzschild is given in Eq. (50).

\section{CONSTRUCTION OF MASTER FUNCTIONS AND EQUATIONS}

The main objective of this section, and also of this work, is to look for the most general master function and equation for both odd- and even-parity modes under the following assumptions:

(1) The perturbative gauge is left completely arbitrary. In this way we can check whether or not the master functions have to be necessarily gauge invariant.

(2) The master function is assumed to be linear in the metric perturbations and its first-order derivatives, as it happens for almost all the known master functions.
The case of the Bardeen-Press master function [77] is an exception since it has been derived along the lines of the Teukolsky [52,53] procedure to decouple perturbations around Kerr but applied to Schwarzschild. In this case, the decoupling follows from using the Newman-Penrose [78] components of the Weyl tensor as master functions. Since the Weyl tensor contains second-order derivatives of the metric perturbations, we cannot recover them from our analysis.

(3) The coefficients in the master function are assumed to be time independent. That is, they only depend on the radial area coordinate $r$. This is expected as those coefficients are built from the Lorentzian metric $g_{a b}$ of the 2D manifold $M^{2}$.

(4) The master function satisfies a wave equation of the form (51). The potential is left arbitrary, in the sense that it will be determined only by the perturbative Einstein equations.

In practical terms, the goal is to decouple the equations for the metric perturbations, the perturbative Einstein field equations [Eq. (6)]. The perturbation in the Einstein tensor, $\delta G_{\mu \nu}=G_{\mu \nu}-\hat{G}_{\mu \nu}$, can be expanded in scalar, vector, and tensor spherical harmonics. For a single $(\ell, m)$ harmonic the structure of $\delta G_{\mu \nu}$ is

$$
\begin{aligned}
& \delta G_{a b}^{\ell m}\left(x^{c}, \Theta^{A}\right)=\mathcal{E}_{a b}^{\ell m}\left(x^{c}\right) Y^{\ell m}\left(\Theta^{A}\right), \\
& \delta G_{a A}^{\ell m}\left(x^{b}, \Theta^{B}\right)= \mathcal{E}_{a}^{\ell m}\left(x^{b}\right) Y_{A}^{\ell m}\left(\Theta^{B}\right)+\mathcal{O}_{a}^{\ell m}\left(x^{b}\right) X_{A}^{\ell m}\left(\Theta^{B}\right), \\
& \delta G_{A B}^{\ell m}\left(x^{a}, \Theta^{C}\right)= \mathcal{E}_{T}^{\ell m}\left(x^{a}\right) T_{A B}^{\ell m}\left(\Theta^{C}\right)+\mathcal{E}_{Y}^{\ell m}\left(x^{a}\right) Y_{A B}^{\ell m}\left(\Theta^{C}\right) \\
&+\mathcal{O}_{X}^{\ell m}\left(x^{a}\right) X_{A B}^{\ell m}\left(\Theta^{C}\right) .
\end{aligned}
$$

We can identify the harmonic components of the perturbative field equations for the even-parity modes, $\left(\mathcal{E}_{a b}^{\ell m}, \mathcal{E}_{a}^{\ell m}, \mathcal{E}_{T}^{\ell m}, \mathcal{E}_{Y}^{\ell m}\right)$, and for the odd-parity modes, $\left(\mathcal{O}_{a}^{\ell m}, \mathcal{O}_{X}^{\ell m}\right)$. Their expressions can be constructed in a straightforward way from the expressions of the perturbations of the Ricci tensor given in Appendix B.

\section{A. Odd-parity (axial) harmonic modes}

In the odd-parity case, we have three independent metric functions, $\left(h_{a}^{\ell m}, h_{2}^{\ell m}\right)$, and the only relevant components of the field equations are $\mathcal{O}_{a}^{\ell m} \mathcal{O}_{X}^{\ell m}$ [see Eqs. (60) and (61)]. The most general master function linear (with coefficients depending only on $r$ ) in the odd-parity metric perturbations and their derivatives is

$$
\begin{aligned}
\Psi_{\mathrm{odd}}^{\ell m}\left(x^{a}\right) & =C_{0}^{\ell}(r) h_{0}^{\ell m}\left(x^{a}\right)+C_{1}^{\ell}(r) h_{1}^{\ell m}\left(x^{a}\right)+C_{2}^{\ell}(r) h_{2}^{\ell m}\left(x^{a}\right) \\
& +C_{3}^{\ell}(r) \dot{h}_{0}^{\ell m}\left(x^{a}\right)+C_{4}^{\ell}(r) h_{0}^{\ell \ell m}\left(x^{a}\right)+C_{5}^{\ell}(r) \dot{h}_{1}^{\ell m}\left(x^{a}\right) \\
& +C_{6}^{\ell}(r) h_{1}^{\prime \ell m}\left(x^{a}\right)+C_{7}^{\ell}(r) \dot{h}_{2}^{\ell m}\left(x^{a}\right)+C_{8}^{\ell}(r) h_{2}^{\ell \ell m}\left(x^{a}\right),
\end{aligned}
$$


where we have used the following simplifying notation for time and radial derivatives:

$$
\dot{\phi}=\frac{\partial \phi}{\partial t}, \quad \phi^{\prime}=\frac{\partial \phi}{\partial r} .
$$

Notice that the coefficients in Eq. (62) only depend on the harmonic number $\ell$. Once we have extracted the different harmonics from the Einstein equations, what is left is a set of linear equations in the perturbations $\left(h_{a}^{\ell m}, h_{2}^{\ell m}\right)$ whose structure is (we drop the harmonic indices to simplify the notation)

$$
\begin{array}{cl}
\mathcal{O}_{t}: & \dot{h}_{1}^{\prime}-h_{0}^{\prime \prime}+\text { LDTs }=0, \\
\mathcal{O}_{r}: & \ddot{h}_{1}-\dot{h}_{0}^{\prime}+\text { LDTs }=0, \\
\mathcal{O}_{X}: & -\frac{1}{f} \ddot{h}_{2}+f h_{2}^{\prime \prime}+\text { LDTs }=0,
\end{array}
$$

where LDTs stands for lower derivative terms (with respect to the other ones), that is, in this particular case they are terms that are linear in the metric perturbations and their first-order derivatives (no second- or higher-order derivatives appear). The first step in the analysis of the general solution to Eqs. (64)-(66) is to study the integrability of Eqs. (64) and (65). Given that they contain $\left(h_{0}^{\prime \prime}, \dot{h}_{1}^{\prime}\right)$ and $\left(\dot{h}_{0}^{\prime}, \ddot{h}_{1}\right)$ respectively, we can study their integrability by differentiating Eq. (64) with respect to $t$ and Eq. (65) with respect to $r$. It turns out that the integrability condition is identically satisfied by using the three equations [Eqs. (64)-(66)]. It is important to mention that to arrive to this conclusion we need to use the fact that the background is a solution of Einstein's field equations, which means to use Eqs. (12) and (13).

The next step in the analysis is to impose that the function in Eq. (62) satisfies the following wave equation (assumption 4):

$$
\square_{2} \Psi_{\text {odd }}\left(x^{a}\right)=\Omega(r) \Psi_{\text {odd }}\left(x^{a}\right),
$$

where $\Omega(r)$ is an arbitrary function of $r$ (and $\ell$ ) that plays the role of the potential [see Eq. (51)]. Given the structure of $\Psi_{\text {odd }}\left(x^{a}\right)$ in Eq. (62), it is clear that the left-hand side of Eq. (67) contains up to third-order derivatives of the metric perturbations $\left(h_{a}, h_{2}\right)$. In this sense, it is important to realize that Eqs. (64)-(66) tell us that from the nine possible second-order derivatives of $\left(h_{a}, h_{2}\right)$, three of them can be written in terms of other second-order derivatives and LDTs. As a consequence, for the twelve possible thirdorder derivatives of $\left(h_{a}, h_{2}\right)$ we have five independent relations between them. ${ }^{3}$ That is, we can write five of the

\footnotetext{
${ }^{3}$ In principle there should be six (two differentiations of three equations), but the integrability condition between Eqs. (64) and (65) eliminates one of them.
}

third-order derivatives of $\left(h_{a}, h_{2}\right)$ in terms of the other ones and LDTs, in an independent way. Therefore, the way to proceed is to expand Eq. (67) and use the expressions for the third-order derivatives that we have just mentioned, together with the expressions that relate the second-order derivatives. After we have used all this information, which comes from the perturbative Einstein equations, we just need to impose the vanishing of the coefficients of the metric perturbations $\left(h_{a}, h_{2}\right)$ and their derivatives. That is, once all the possible information coming from Einstein's equations is used, the remainder has to vanish for Eq. (67) to hold. Once we have completed this process, we should have obtained the most general odd-parity master function, together with the allowed potential(s).

In our study, the second-order derivatives of the metric perturbations that we are going to eliminate are $\ddot{h}_{1}, \dot{h}_{1}^{\prime}$, and $\ddot{h}_{2}$. In addition, we also eliminate the third-order derivatives that can be computed from these second-order derivatives, i.e., $\breve{h}_{1}, \ddot{h}_{1}^{\prime} \dot{h}_{1}^{\prime \prime}, \breve{h}_{2}$, and $\ddot{h}_{2}^{\prime}$. After eliminating all these derivatives we arrive to an expression of the form

$$
\begin{aligned}
\square_{2} \Psi_{\text {odd }}= & \tau_{0} \dddot{h}_{0}+\tau_{1} \ddot{h}_{0}^{\prime}+\tau_{2} \dot{h}_{0}^{\prime \prime}+\tau_{3} h_{0}^{\prime \prime \prime}+\tau_{4} h_{1}^{\prime \prime \prime} \\
& +\tau_{5} \ddot{h}_{0}+\tau_{6} \dot{h}_{0}^{\prime}+\tau_{7} \dot{h}_{0}^{\prime \prime}+\tau_{8} \dot{h}_{1}^{\prime \prime}+\tau_{9} \dot{h}_{2}^{\prime}+\tau_{10} h_{2}^{\prime \prime} \\
& +\tau_{11} \dot{h}_{0}+\tau_{12} h_{0}^{\prime}+\tau_{13} \dot{h}_{1}+\tau_{14} h_{1}^{\prime}+\tau_{15} \dot{h}_{2} \\
& +\tau_{16} h_{2}^{\prime}+\tau_{17} h_{0}+\tau_{18} h_{1}+\tau_{19} h_{2} .
\end{aligned}
$$

Notice that there are no third-order derivatives of $h_{2}$. The explanation is that Eq. (66) can be rewritten as

$$
-\frac{1}{2} \square_{2} h_{2}+\text { LDTs }=0 .
$$

That is, $\square_{2} h_{2}$ only produces LDTs and this is why there are no third-order derivatives of $h_{2}$ in Eq. (68). Now that only independent derivatives of the metric perturbations appear [Eq. (68)] we can proceed to analyze the consequences of the vanishing of their coefficients. To begin with, the vanishing of $\tau_{0}$ implies

$$
C_{3}=0 \text {. }
$$

The vanishing of $\tau_{4}$ leads to

$$
C_{6}=0 \text {. }
$$

It turns out that Eq. (70) and Eq. (71) imply that $\tau_{2}=0$, so there are no extra conditions coming from this term. The vanishing of $\tau_{1}$ and $\tau_{3}$ lead to the same condition

$$
C_{5}=-C_{4}
$$

This exhausts the information coming from the vanishing of the coefficients of the third-order derivatives of the 
metric perturbations. Let us now look at the coefficients of the second-order derivatives. We assume that Eqs. (70)(72) hold. The vanishing of the coefficients $\tau_{6}$ and $\tau_{8}$ yields only one condition (they are equivalent):

$$
C_{8}=-\frac{1}{2} C_{1} .
$$

Introducing this into the equation coming from the vanishing of the coefficient $\tau_{10}$, we obtain an equation for $C_{1}(r)$

$$
C_{1}^{\prime}+\left(\frac{1}{r}-\frac{f^{\prime}}{f}\right) C_{1}=0 \Rightarrow C_{1}(r)=\frac{K_{1} f(r)}{r},
$$

where $K_{1}$ is an arbitrary constant. Similarly, the vanishing of the coefficient $\tau_{9}$ implies the following equation for $C_{7}(r)$ :

$$
r C_{7}^{\prime}+C_{7}=0 \Rightarrow C_{7}(r)=\frac{K_{7}}{r}
$$

where $K_{7}$ is another arbitrary constant. The coefficients $\tau_{5}$ and $\tau_{7}$ contain the same information. Their vanishing allows us to obtain an expression for $C_{4}(r)$ :

$$
C_{4}=-\frac{r}{2}\left(C_{0}+2 C_{7}\right) \Rightarrow C_{4}=-\frac{r}{2}\left(C_{0}+\frac{2 K_{7}}{r}\right) \text {. }
$$

And this exhausts the information coming from the vanishing of the coefficients of the second-order derivatives of the metric perturbations. The analysis of the consequences of the other terms involve the right-hand side of Eq. (67), i.e., the potential. Then, let us analyze the coefficients of the first-order derivatives. To begin with, since $C_{3}(r)$ vanishes [Eq. (70)], the coefficient of $\dot{h}_{0}, \tau_{11}$ does not involve $\Omega(r)$. It actually provides an expression for $C_{2}(r)$ :

$$
C_{2}(r)=\frac{C_{1}(r)}{r}=\frac{K_{1} f(r)}{r^{2}} .
$$

The coefficient $\tau_{14}$ vanishes if we introduce this expression for $C_{2}(r)$. From the vanishing of the coefficient $\tau_{15}$ we obtain an expression for the derivative of $C_{0}(r)($ for $\ell \neq 1)$

$$
C_{0}^{\prime}=\frac{2 K_{7}}{(\ell+2)(\ell-1)}\left(\Omega-\frac{f^{\prime}}{r}\right)
$$

where we have used Eq. (13) for the background, i.e., for $f(r)$. On the other hand, the information in the coefficients $\tau_{12}$ and $\tau_{13}$ is the same. It is a relationship between the coefficient $C_{0}(r)$ and its first- and second-order derivatives:

$C_{0}^{\prime \prime}=\left(\frac{2}{r}-\frac{f^{\prime}}{f}\right) C_{0}^{\prime}+\frac{\Omega-\Omega_{*}}{f}\left(C_{0}+\frac{2 K_{7}}{r}\right)-\frac{2 K_{7} f^{\prime}}{r^{2} f}$, where we have introduced the following definition:

$$
\begin{aligned}
\Omega_{*}(r) & =\Lambda+\frac{1}{r^{2}}\left[\ell(\ell+1)+r\left(r f^{\prime}\right)^{\prime}+2(f-1)\right] \\
& =\frac{(\ell+2)(\ell-1)+2 f-r f^{\prime}}{r^{2}} \\
& =\Lambda+\frac{\ell(\ell+1)+3(f-1)}{r^{2}},
\end{aligned}
$$

where the different equalities appear as a consequence of using the equations for $f(r)$ [Eqs. (12) and (13)]. By using Eq. (78) we can eliminate $C_{0}^{\prime}(r)$ from Eq. (79) and obtain the following alternative expression for $C_{0}^{\prime \prime}(r)$ :

$$
\begin{aligned}
C_{0}^{\prime \prime}(r)= & \frac{\Omega-\Omega_{*}}{f} C_{0} \\
& +\frac{2 K_{7} r \Omega_{*}}{(\ell+2)(\ell-1) f}\left(\Omega-\Omega_{*}+2 \frac{f-r f^{\prime}}{r^{2}}\right) .
\end{aligned}
$$

Actually, we can also rewrite the equation for $C_{0}^{\prime}(r)$ [Eq. (78)] as

$$
C_{0}^{\prime}=\frac{2 K_{7}}{(\ell+2)(\ell-1)}\left(\Omega-\Omega_{*}+o_{0}\right)
$$

where

$$
o_{0}(r)=\Omega_{*}(r)-\frac{f^{\prime}(r)}{r} .
$$

We can integrate the equation for $C_{0}^{\prime}(r)$ to get

$$
\begin{aligned}
C_{0}(r)= & K_{0}+\frac{2 K_{7}}{(\ell+2)(\ell-1)} \\
& \times\left\{\frac{f(r)-1-\Lambda r^{2}}{2 r}+\int_{r} d r^{\prime} \Omega\left(r^{\prime}\right)\right\},
\end{aligned}
$$

where $K_{0}$ is an integration constant. After all this, the only coefficient of the first-order derivatives of the metric perturbations left to be analyzed is $\tau_{16}$. If we introduce the expression of $C_{2}(r)$ [Eq. (77)] into the coefficient $\tau_{16}$ and impose its vanishing we arrive at the following relation:

$$
K_{1}\left[\Omega(r)-\Omega_{*}(r)\right]=0,
$$

This equation constitutes a bifurcation point in our analysis. Either $K_{1}=0 \Rightarrow C_{1}(r)=0$ or we have an expression for the potential $\Omega(r)$ in terms of $r$ and $\ell$ [this expression is given in Eq. (80)]. Therefore, there are two branches of possible solutions to the problem we posed at the beginning of this section.

At this point, we only have to focus on the coefficients of the metric perturbations themselves, i.e., $\left(\tau_{17}, \tau_{18}, \tau_{19}\right)$, and the consistency between the expressions for the first- and 
second-order derivatives of $C_{0}(r)$ [Eqs. (82) and (81) respectively]. The equation coming from $\tau_{17}$ does not provide new information, in the sense that it becomes a trivial identity $0=0$ if we use the previous information. The coefficients $\tau_{18}$ and $\tau_{19}$, after using the previous information and the equations for the background, both lead to Eq. (85). Therefore, the only thing left is to analyze the compatibility between Eqs. (82) and (81). This can be done by taking the derivative of $C_{0}^{\prime}(r)$ by using Eq. (82) and subtract $C_{0}^{\prime \prime}(r)$ from it by using Eq. (81). In the case they were compatible we should be able to reduce the subtraction to an identity $0=0$ by using all the previous information. Otherwise, we should obtain new information/ conditions on our unknowns. This is indeed what happens and the new information is encoded in the following equation:

$$
\begin{aligned}
& {\left[\Omega(r)-\Omega_{*}(r)\right] C_{0}(r)+\frac{2 K_{7}}{(\ell+2)(\ell-1)}} \\
& \quad \times\left[o_{1}(r)\left(\Omega(r)-\Omega_{*}(r)\right)^{\prime}+o_{2}(r)\left(\Omega(r)-\Omega_{*}(r)\right)\right. \\
& \left.\quad+o_{3}(r)\right]=0,
\end{aligned}
$$

where

$$
\begin{gathered}
o_{1}=-f, \\
o_{2}=r \Omega_{*}, \\
o_{3}=2\left(\frac{f}{r}-f^{\prime}\right) \Omega_{*}-f\left(\Omega_{*}^{\prime}-3 \frac{f-1}{r^{3}}-\frac{\Lambda}{r}\right) .
\end{gathered}
$$

At this point, we have to deal with Eqs. (85) and (86), taking into account that in this analysis $r$ is arbitrary and $\ell$ is an integer number. Another important point to consider is that $o_{3}(r)$ is in general not zero (it does not vanish everywhere). Therefore, $\Omega(r)=\Omega_{*}(r)$ would imply $K_{7}=0$.

On the other hand, Eq. (86), in the case $\Omega(r) \neq \Omega_{*}(r)$, can in principle provide an expression for the coefficient $C_{0}(r)$. To that end, we must first make sure that this equation is compatible with the equations for the derivatives of $C_{0}(r)$ [Eqs. (82) and (81)]. Given that Eq. (86) is the compatibility between $C_{0}^{\prime}$ and $C_{0}^{\prime \prime}$, we just need to check the compatibility with $C_{0}^{\prime}$ [Eq. (82)]. To do so, we can take the derivative of Eq. (86) and compare it with Eq. (82). Actually, if we use Eq. (82) to eliminate $C_{0}^{\prime}$ from the derivative of Eq. (86) we obtain a new relation that has a form very similar to Eq. (86):

$$
\Gamma[r, \Omega] C_{0}(r)+K_{7} \Delta[r, \Omega]=0,
$$

where $\Gamma[r, \Omega]$ and $\Delta[r, \Omega]$ are functionals of $\Omega(r)$. From these two relations [Eqs. (86) and (90)] we can eliminate either $C_{0}(r)$ or $K_{7}$. In any case, and assuming that
$\Omega(r) \neq \Omega_{*}(r)$, we obtain an equation for $\Omega^{\prime \prime}(r)$ that has the following form:

$$
\begin{aligned}
& K_{7}\left[\left(\hat{o}_{1}(r) \frac{\delta \Omega^{\prime}(r)}{\delta \Omega(r)}\right)^{\prime}+\left(\frac{\hat{o}_{3}(r)}{\delta \Omega(r)}\right)^{\prime}+\hat{o}_{o}(r)+\hat{o}_{2}^{\prime}(r)-\delta \Omega(r)\right] \\
& \quad=0
\end{aligned}
$$

where we have introduced the following definitions:

$$
\begin{gathered}
\delta \Omega(r)=\Omega(r)-\Omega_{*}(r), \\
\hat{o}_{I}(r)=-o_{I}(r)(I=0-3) .
\end{gathered}
$$

In this way, $\hat{o}_{1}(r)=f(r)$. From Eq. (91), it is clear that if $K_{7} \neq 0$, we have an equation for $\Omega^{\prime \prime}(r)$ which is nonlinear in $\Omega(r)$.

In principle, we can differentiate Eq. (90) and we would get an expression of the same form by using again Eq. (82) for $C_{0}^{\prime}(r)$. Combining the new equation with Eq. (86) we can again eliminate either $C_{0}(r)$ or $K_{7}$. This would provide us with a new equation where the only unknown is the potential $\Omega(r)$. It turns out that using the equation for $\Omega^{\prime \prime}(r)$ provided by Eq. (91), we get an identity $0=0$, and this ends the chain of possible equations of the form in Eq. (90).

In summary, we end up with two different branches. The first branch is determined by the following relation:

$$
\Omega(r)=\Omega_{*}(r) \quad \Rightarrow \quad \delta \Omega(r)=0 .
$$

Then, the potential for odd-parity perturbations, $\Omega_{*}(r)$, is given by Eq. (80). In the case of a Schwarzschild background, this potential has been shown in Eq. (57), while for the case of a de Sitter background, it is simply the centrifugal barrier (the same one as in a Minkowski, and also anti-de Sitter, background):

$$
\Omega_{*}^{\mathrm{dS}}(r)=\frac{\ell(\ell+1)}{r^{2}} .
$$

In this first branch, Eq. (85) is automatically satisfied. On the other hand, Eq. (86) implies:

$$
K_{7}=0 \text {. }
$$

Therefore, according to Eq. (82), the coefficient $C_{0}(r)$ is constant:

$$
C_{0}^{\prime \prime}(r)=C_{0}^{\prime}(r)=0 \quad \Rightarrow \quad C_{0}(r)=K_{0},
$$

where $K_{0}$ is a constant. This is also compatible with the equation for $C_{0}^{\prime \prime}(r)$ [Eq. (81)]. Then, the most general oddparity master function in this branch is 


$$
\begin{aligned}
\Psi_{\text {odd }}(t, r)= & \frac{r K_{0}}{2}\left(\dot{h}_{1}(t, r)-h_{0}^{\prime}(t, r)\right)+K_{0} h_{0}(t, r) \\
& +K_{1} \frac{f(r)}{r} h_{1}(t, r) \\
& +\frac{K_{1} f(r)}{r}\left(\frac{1}{r} h_{2}(t, r)-\frac{1}{2} h_{2}^{\prime}(t, r)\right) .
\end{aligned}
$$

Using the definitions introduced in Sec. IIE we can rewrite this master function in a completely covariant way:

$$
\Psi_{\mathrm{odd}}(t, r)=K_{1} \Psi_{\mathrm{RW}}(t, r)-\frac{(\ell+2)(\ell-1)}{4} K_{0} \Psi_{\mathrm{CPM}}(t, r) .
$$

That is, the most general master function in the first branch is a linear combination of the Regge-Wheeler and Cunningham-Price-Moncrief master functions [see Eqs. (46) and (47) respectively]. Therefore, it is covariant and gauge invariant by construction. It is important here to note that the Regge-Wheeler master function turns out to be the time derivative of the Cunningham-Price-Moncrief master function [48]:

$$
t^{a} \Psi_{\mathrm{CPM}: a}=2 \Psi_{\mathrm{RW}}
$$

Finally, the potential corresponds to the already known potential, namely the Regge-Wheeler one. This ends the analysis of the first branch.

Let us now consider the second branch. It is characterized by $\Omega(r) \neq \Omega_{*}(r)$ (i.e., $\delta \Omega(r) \neq 0$ ) and hence, we must necessarily have

$$
K_{1}=0
$$

and $\delta \Omega(r)$ has to satisfy Eq. (91). In this case, the only nonzero coefficients of $\Psi_{\text {odd }}$ are $C_{0}(r)$ [Eq. (84)], $C_{4}(r)$ [Eq. (76)], $C_{5}(r)$ [=- $\left.C_{4}(r)\right]$, and $C_{7}(r)$ [Eq. (75)]. Introducing these expressions we can write the most general master function in the second branch as

$$
\begin{aligned}
\Psi_{\text {odd }}(t, r)= & -\frac{(\ell+2)(\ell-1)}{4}\left(K_{0}+\hat{K}_{7} \Xi(r)\right) \Psi_{\mathrm{CPM}}(t, r) \\
& +K_{7} \Phi_{\mathrm{ON}}(t, r),
\end{aligned}
$$

where

$$
\begin{gathered}
\hat{K}_{7}=\frac{2 K_{7}}{(\ell+2)(\ell-1)}, \\
\Xi(r)=\frac{f(r)-1-\Lambda r^{2}}{2 r}+\int_{r} d r^{\prime} \Omega\left(r^{\prime}\right),
\end{gathered}
$$

and $\Phi_{\mathrm{ON}}(t, r)$ is a new odd-parity function that can be given in a completely covariant form as

$$
\Phi_{\mathrm{ON}}(t, r)=\varepsilon^{a b} \tilde{h}_{a: b} .
$$

One can check that $\Phi_{\mathrm{ON}}(t, r)$ is also a gauge-invariant quantity although it is not by itself a master function. However, the combination with $\Psi_{\mathrm{CPM}}(t, r)$ that appears in Eq. (102), whose coefficient is $K_{7}$, is an odd-parity master function. Then, the most general odd-parity master function in the second branch, Eq. (102), is fully covariant and gauge invariant. The potential in this second branch is any function satisfying the nonlinear ODE of Eq. (91). Regarding this equation, it is worth noting that if we write it in terms of $\delta V=f \delta \Omega$ [see Eq. (56)], use the expressions for $\hat{o}_{o}$ and $\hat{o}_{2}$, which satisfy

$$
\hat{o}_{o}+\hat{o}_{2}^{\prime}-f^{\prime \prime}=0,
$$

and exchange derivatives with respect to $r$ with derivatives with respect to the tortoise coordinate we arrive at the following simpler equation:

$$
\left(\frac{\delta V_{, x}}{\delta V}\right)_{, x}+2\left(\frac{V_{, x}^{\text {odd }}}{\delta V}\right)_{, x}-\delta V=0
$$

where $V^{\text {odd }}=f \Omega_{*}$ is the Regge-Wheeler potential. Finally, it is important to remark that changing the potential we are changing at the same time the master function. This ends the analysis of the odd-parity case.

\section{B. Even-parity (polar) harmonic modes}

In the even-parity case (polar perturbations) we have seven independent metric functions, $\left(h_{a b}^{\ell m}, \mathrm{~J}_{a}^{\ell m}, K^{\ell m}, G^{\ell m}\right)$, and this time we have seven relevant field equations, coming from the components $\left(\mathcal{E}_{a b}^{\ell m}, \mathcal{E}_{a}^{\ell m}, \mathcal{E}_{T}^{\ell m}, \mathcal{E}_{Y}^{\ell m}\right)$ of the perturbative field equations. Taking into account the assumptions we imposed on the master function before, the most general ansatz to start with is ${ }^{4}$

$$
\begin{aligned}
\Psi_{\mathrm{even}}^{\ell m}= & C_{0}^{\ell} h_{00}^{\ell m}+C_{1}^{\ell} h_{01}^{\ell m}+C_{2}^{\ell} h_{11}^{\ell m}+C_{3}^{\ell} \mathrm{J}_{0}^{\ell m} \\
& +C_{4}^{\ell} \mathrm{J}_{1}^{\ell m}+C_{5}^{\ell} K^{\ell m}+C_{6}^{\ell} G^{\ell m}+C_{7}^{\ell} \dot{h}_{00}^{\ell m}+C_{8}^{\ell} h_{00}^{\ell \ell m} \\
& +C_{9}^{\ell} \dot{h}_{01}^{\ell m}+C_{10}^{\ell} h_{01}^{\ell \ell m}+C_{11}^{\ell} \dot{h}_{11}^{\ell m}+C_{12}^{\ell} h_{11}^{\ell \ell m}+C_{13}^{\ell} \mathbf{J}_{0}^{\ell m} \\
& +C_{14}^{\ell} \mathrm{J}_{0}^{\prime \ell m}+C_{15}^{\ell} \dot{\mathrm{j}}_{1}^{\ell m}+C_{16}^{\ell} \mathrm{J}_{1}^{\prime \ell m}+C_{17}^{\ell} \dot{K}^{\ell m}+C_{18}^{\ell} K^{\prime \ell m} \\
& +C_{19}^{\ell} \dot{G}^{\ell m}+C_{20}^{\ell} G^{\ell \ell m} .
\end{aligned}
$$

For the sake of simplicity we have hidden the dependence of the different functions since it is clear that the coefficients $C_{I}^{\ell}(I=0, \ldots, 20)$ only depend on the radial

\footnotetext{
${ }^{4}$ As in the odd-parity case, we use coefficients named $C_{I}^{\ell}$ but no confusion should arise since they are purely auxiliary quantities and there are no cross references.
} 
coordinate $r$, and the metric perturbations $\left(h_{a b}^{\ell m}, \mathrm{~J}_{a}^{\ell m}, K^{\ell m}\right.$, $G^{\ell m}$ ) depend on the coordinates of $M^{2}$, i.e., $\left\{x^{a}\right\}$. Following the procedure of the odd-parity case, let us analyze the structure of the perturbative field equations for the metric perturbations $\left(h_{a b}^{\ell m}, \mathbf{J}_{a}^{\ell m}, K^{\ell m}, G^{\ell m}\right)$ This essentially means to analyze the structure of the terms containing second-order derivatives. Dropping again the harmonic indices, we find that the equations of interest have the following form:

$$
\begin{gathered}
\mathcal{E}_{t t}: K^{\prime \prime}+\mathrm{LDTs}=0, \\
\mathcal{E}_{t r}: \dot{K}^{\prime}+\mathrm{LDTs}=0, \\
\mathcal{E}_{r r}: \ddot{K}+\mathrm{LDTs}=0, \\
\mathcal{E}_{t Y}: \dot{\mathrm{J}}_{1}^{\prime}-\dot{\mathrm{J}}_{0}^{\prime \prime}+\mathrm{LDTs}=0, \\
\mathcal{E}_{r Y}: \ddot{\mathrm{J}}_{1}-\dot{\mathrm{J}}_{1}^{\prime}+\mathrm{LDTs}=0, \\
\mathcal{E}_{T}:-\frac{1}{f} \ddot{K}+f K^{\prime \prime}-h_{00}^{\prime \prime}+2 \dot{h}_{01}^{\prime}-\ddot{h_{11}}+\mathrm{LDTs}=0, \\
\mathcal{E}_{Y}:-\frac{1}{f} \ddot{G}+f G^{\prime \prime}+\mathrm{LDTs}=0 .
\end{gathered}
$$

Given that Eqs. (112) and (113) contain $\left(\mathrm{J}_{0}^{\prime \prime}, \dot{\mathrm{J}}_{1}^{\prime}\right)$ and $\left(\dot{\mathrm{J}}_{0}^{\prime}, \ddot{\mathrm{J}}_{1}\right)$ respectively, we can study their integrability by differentiating Eq. (112) with respect to $t$ and Eq. (113) with respect to $r$. The result is that this integrability condition is identically satisfied by using the following equations: (109), (111), (113), (114), and (115). Like in the oddparity case, in order to arrive to this conclusion we have used the equations for the background metric function $f(r)$. Similarly, Eqs. (109)-(111) contain all the second-order derivatives of $K$, i.e., $\left(\ddot{K}, \dot{K}^{\prime}, K^{\prime \prime}\right)$. One can show that their integrability conditions are satisfied by using the other equations and that the background is a solution of Einstein's equations. The fact that the integrability conditions are satisfied, both for odd- and even-parity perturbations, is intimately related with the metric perturbations satisfying a linearized version of the (contracted) second Bianchi identities: $\bar{g}^{\rho \mu} \bar{\nabla}_{\rho} \delta G_{\mu \nu}=0$. On the other hand, we can substitute the expressions for the second-order derivatives of $K$ [Eqs. (109)-(111)] into Eq. (114) so that it becomes a relation between second-order derivatives of $h_{a b}$.

Like in the odd-parity case, we impose our master function candidate in Eq. (108) to satisfy a wave equation of the type

$$
\square_{2} \Psi_{\text {even }}\left(x^{a}\right)=\Omega(r) \Psi_{\text {even }}\left(x^{a}\right)
$$

where $\Omega(r)$ is a function of $r$ (and $\ell$ ) to be determined and that will play the role of the potential for the dynamics of even-parity perturbations. When we insert the general even-parity master function of Eq. (108) we will get again a linear combination of the metric perturbations $\left(h_{a b}^{\ell m}, \mathbf{J}_{a}^{\ell m}, K^{\ell m}, G^{\ell m}\right)$ and their derivatives up to third order. However, not all these derivatives are independent since Eqs. (109)-(115) already determine a subset of secondorder derivatives (seven of them), and hence a subset of the third-order derivatives too. To be more specific, let us make a choice. From the perturbative Einstein equations we determine the following second-order derivatives: $\ddot{h}_{11}, \ddot{\mathrm{J}}_{1}$, $\dot{\mathrm{J}}_{1}^{\prime}, \ddot{K}, \dot{K}^{\prime}, K^{\prime \prime}$, and $\ddot{G}$. We can then substitute these derivatives into Eq. (116). In addition, we can also substitute the third-order derivatives that can be estimated from them, namely, $\dddot{h}_{11}, \ddot{h}_{11}^{\prime}, \dddot{\mathrm{J}}_{1}, \ddot{\mathrm{J}}_{1}^{\prime}, \dot{\mathrm{J}}_{1}^{\prime \prime}, \dddot{K}, \ddot{K}^{\prime}, \dot{K}^{\prime \prime}, K^{\prime \prime \prime}, \dddot{G}$, and $\ddot{G}^{\prime}$. Once this is done we arrive to an expression of the form ${ }^{5}$

$$
\begin{aligned}
\square_{2} \Psi_{\text {even }}= & \tau_{0} \dddot{h}_{00}+\tau_{1} \ddot{h}_{00}^{\prime}+\tau_{2} \dot{h}_{00}^{\prime \prime}+\tau_{3} h_{00}^{\prime \prime \prime}+\tau_{4} \dddot{h}_{01}+\tau_{5} \ddot{h}_{01}^{\prime}+\tau_{6} \dot{h}_{01}^{\prime \prime}+\tau_{7} h_{01}^{\prime \prime \prime}+\tau_{8} \dot{h}_{11}^{\prime \prime}+\tau_{9} h_{11}^{\prime \prime \prime}+\tau_{10} \dddot{\mathrm{J}}_{0}+\tau_{11} \ddot{\mathrm{J}}_{0}^{\prime}+\tau_{12} \dot{\mathrm{J}}_{0}^{\prime \prime} \\
& +\tau_{13} \mathrm{~J}_{0}^{\prime \prime \prime}+\tau_{14} \mathrm{~J}_{1}^{\prime \prime \prime}+\tau_{15} \ddot{h}_{00}+\tau_{16} \dot{h}_{00}^{\prime}+\tau_{17} \dot{h}_{00}^{\prime \prime}+\tau_{18} \ddot{h}_{01}+\tau_{19} \dot{h}_{01}^{\prime}+\tau_{20} \dot{h}_{01}^{\prime \prime}+\tau_{21} \dot{h}_{11}^{\prime}+\tau_{22} \dot{h}_{11}^{\prime \prime}+\tau_{23} \ddot{\mathrm{J}}_{0}+\tau_{24} \dot{\mathrm{J}}_{0}^{\prime} \\
& +\tau_{25} \dot{\mathrm{J}}_{0}^{\prime \prime}+\tau_{26} \dot{\mathrm{J}}_{1}^{\prime \prime}+\tau_{27} \dot{G}_{0}^{\prime}+\tau_{28} G_{0}^{\prime \prime}+\tau_{29} \dot{h}_{00}+\tau_{30} h_{00}^{\prime}+\tau_{31} \dot{h}_{01}+\tau_{32} h_{01}^{\prime}+\tau_{33} \dot{h}_{11}+\tau_{34} h_{11}^{\prime}+\tau_{35} \dot{\mathrm{J}}_{0}+\tau_{36} \dot{\mathrm{J}}_{0}^{\prime} \\
& +\tau_{37} \dot{\mathrm{J}}_{1}+\tau_{38} \dot{\mathrm{J}}_{1}^{\prime}+\tau_{39} \dot{K}+\tau_{40} K^{\prime}+\tau_{41} \dot{G}+\tau_{42} G^{\prime}+\tau_{43} h_{00}+\tau_{44} h_{01}+\tau_{45} h_{11}+\tau_{46} \mathrm{~J}_{0}+\tau_{47} \mathrm{~J}_{1}+\tau_{48} K+\tau_{49} G .
\end{aligned}
$$

As it already happened in the odd-parity case with the metric perturbation $h_{2}$, here there are no third-order derivatives of $G$, and it is due to the same reason. If we look at Eq. (115), it turns out it can be rewritten, up to a

\footnotetext{
${ }^{5}$ As in the odd-parity case, we use coefficients named $\tau_{I}$ but no confusion should arise since they are purely auxiliary quantities and there are no cross references.
}

numerical factor, as $\square_{2} G+\mathrm{LDTs}=0$. And since the operator $\square_{2}$ only produces LDTs, no third derivatives of $G$ appear in Eq. (117).

Let us now analyze the implications of the vanishing of the coefficients $\tau_{I}(I=0, \ldots, 49)$ for the general form of the even-parity master function. To begin with, the vanishing of the coefficients $\tau_{0}, \ldots, \tau_{5}$ implies the vanishing of the following coefficients of the master function [see Eq. (108)]: 


$$
C_{7}=C_{8}=C_{9}=C_{10}=C_{11}=C_{12}=0 .
$$

We have arrived at this result by using the information from one coefficient into the next one. Notice that this implies that the master function cannot contain any first-order derivatives of the metric perturbations $h_{a b}$. Using this information, the vanishing of the coefficients $\tau_{6}, \ldots, \tau_{9}$ does not provide any additional information. The vanishing of $\tau_{10}$ and $\tau_{12}$ implies

$$
C_{13}=C_{16}=0 .
$$

Taking this into account, the coefficient $\tau_{14}$ does not provide any additional information. The coefficients $\tau_{11}$ and $\tau_{13}$ contain the same information:

$$
C_{14}+C_{15}=0
$$

The vanishing of the coefficient $\tau_{15}$ tells us that

$$
C_{0}=0 \text {. }
$$

The coefficient $\tau_{16}$, together with the previous information, leads to

$$
C_{17}=\frac{r}{f} C_{14}
$$

Similarly, from the coefficient $\tau_{17}$ we have

$$
C_{18}=-\frac{r}{f} C_{2}
$$

From $\tau_{18}$ we get

$$
C_{14}=-C_{1} .
$$

Using all the information obtained up to now, we can see that the coefficients $\tau_{19}, \ldots, \tau_{22}$ do not provide new information. Instead, from $\tau_{23}$ we get an expression for $C_{19}$ in terms of $C_{1}$ and $C_{3}$ :

$$
C_{19}=\frac{2 f-\ell(\ell+1)}{2 f} r C_{1}-\frac{r^{2}}{2} C_{3}
$$

From $\tau_{24}$ we obtain a similar expression for $C_{20}$

$$
C_{20}=-\frac{\ell(\ell+1)}{2 f} r C_{2}-\frac{r^{2}}{2} C_{4}
$$

With this, the conditions coming from $\tau_{25}$ and $\tau_{26}$ are automatically satisfied. The coefficient $\tau_{27}$ leads to a simple ODE:

$$
r C_{19}^{\prime}-C_{19}=0
$$

Taking into account Eq. (125) we can solve the equation for $C_{3}$,

$$
C_{3}=\frac{K_{13}}{r}+\frac{2 f-\ell(\ell+1)}{r f} C_{1},
$$

where $K_{13}$ is an integration constant. After substitution in Eq. (125), the coefficient $C_{19}$ takes the following simple form [compatible with Eqs. (125) and (127)]:

$$
C_{19}(r)=-\frac{K_{13} r}{2}
$$

Similarly, from $\tau_{28}$ we can obtain an ODE from which we find an expression for $C_{4}$

$$
C_{4}=K_{24} \frac{f}{r}-\frac{\ell(\ell+1)}{r f} C_{2},
$$

where $K_{24}$ is another integration constant. And thanks to this, the coefficient $C_{20}$ takes the following simple form:

$$
C_{20}(r)=-\frac{K_{24}}{2} r f(r)
$$

Using the previous expression, the condition coming from $\tau_{29}$ determines the coefficient $C_{1}$ :

$$
C_{1}=\frac{K_{13} f}{\ell(\ell+1)+r f^{\prime}-2 f}
$$

and the coefficient $\tau_{30}$ determines the coefficient $C_{5}$ :

$C_{5}=-\frac{K_{24}}{2}+\frac{(\ell+2)(\ell-1)+3 r f^{\prime}+2 \Lambda r^{2}}{2 f^{2}} C_{2}$.

With all this information the relations coming from the coefficients $\tau_{31}, \ldots, \tau_{34}$ are satisfied. The coefficient $\tau_{35}$ provides an expression for $C_{6}$ :

$C_{6}=\frac{\ell(\ell+1)}{4}\left(\frac{(\ell+2)(\ell-1)+3 r f^{\prime}+2 \Lambda r^{2}}{f^{2}} C_{2}-K_{24}\right)$.

To sum up the situation until now: All the nonzero coefficients are ultimately found either in terms of $C_{1}$ or $C_{2}$. The coefficient $C_{1}$ has already been determined in terms of $r, f$, and an integration constant, $K_{13}$ [see Eq. (132)]. Only $C_{2}$ and the potential $\Omega$ have to be determined from the equations imposed by the rest of coefficients $\tau_{I}$. In what follows we use all the information found until now.

The coefficient $\tau_{38}$ brings no new information. The equation coming from the coefficient $\tau_{36}$ can be written as follows: 


$$
K_{13}\left[\Omega(r)-\Omega_{*}(r)\right]=0,
$$

where $\Omega_{*}(r)$ is a given function of $r, f$ (and its derivatives), $\ell$, and $\Lambda$. As in the odd-parity case [see Eq. (85)], this equation is also a branch point in the analysis. By doing some algebra (and using the field equations of the background spacetime) we can write $\Omega_{*}(r)$ as follows:

$$
\Omega_{*}(r)=\frac{\lambda^{3}(r)-2 \Lambda r^{2}[\lambda(r)-(\ell+2)(\ell-1)]^{2}+2(\ell+2)^{2}(\ell-1)^{2}\left(\ell^{2}+\ell+1\right)}{3 r^{2} \lambda^{2}(r)},
$$

where $\lambda(r)$ was defined in Eq. (49). The relations coming from the vanishing of the coefficients $\tau_{37}, \tau_{39}, \tau_{41}, \tau_{44}$, and $\tau_{46}$ also reduce to the bifurcation point represented by Eq. (135). To get there we have used sometimes the equations for the background metric [Eqs. (12) and (13)]. On the other hand, from the vanishing of the coefficient $\tau_{40}$ we arrive at an expression for the secondorder derivative of the coefficient $C_{2}$ :

$$
C_{2}^{\prime \prime}=e_{1} C_{2}^{\prime}+e_{2} C_{2}+K_{24} \frac{f}{r^{2}},
$$

where the coefficients $e_{1}(r)$ and $e_{2}(r)$, using the field equations of the background [Eqs. (12) and (13)], are

$$
\begin{aligned}
& e_{1}=\frac{\ell(\ell+1)-3\left(\Lambda r^{2}-1\right)-f}{r f}, \\
& e_{2}=\frac{f^{2}+\left(1-5 \Lambda r^{2}\right) f+2\left(r^{2} \Lambda-1\right)\left(\ell^{2}+\ell+2-2 \Lambda r^{2}\right)}{r^{2} f^{2}} \\
& +\frac{\Omega}{f} .
\end{aligned}
$$

As we can see, the function $e_{2}(r)$ contains the potential $\Omega(r)$. On the other hand, from the coefficient $\tau_{42}$ we arrive at an expression for the first derivative of the coefficient $C_{2}$ of the form

$$
C_{2}^{\prime}=e_{4} C_{2}+K_{24} e_{3}
$$

where the coefficients $e_{3}(r)$ and $e_{4}(r)$ are

$$
\begin{gathered}
e_{3}=-f^{2} \frac{r^{2} \Omega+\lambda-(\ell+2)(\ell-1)}{\ell(\ell+1) r \lambda}, \\
e_{4}=\left(\ln \frac{r f^{2}}{\lambda}\right)^{\prime} .
\end{gathered}
$$

Here, the function $e_{3}(r)$ is the one that contains the potential $\Omega(r)$. It turns our that Eq. (140) can be integrated to obtain the following expression for $C_{2}(r)$ :

$$
\begin{aligned}
C_{2}(r)= & \frac{r f^{2}(r)}{\lambda(r)}\left\{K_{2}+\frac{K_{24}}{\ell(\ell+1)}\left[\frac{\lambda(r)-(\ell+2)(\ell-1)}{2 r}\right.\right. \\
& \left.\left.-\int_{r} d r^{\prime} \Omega\left(r^{\prime}\right)\right]\right\} .
\end{aligned}
$$

where $K_{2}$ is another integration constant.

Going back to the analysis of the $\tau_{I}$ coefficients, using previous information [including the equations for $C_{2}^{\prime}$, Eq. (140), and $C_{2}^{\prime \prime}$, Eq. (137)] and some algebra, it is possible to see that the equations coming from the vanishing of the coefficients $\tau_{43}, \tau_{45}, \tau_{47}, \tau_{48}$, and $\tau_{49}$ are identically satisfied. This exhausts all the information coming from the coefficients $\tau_{I}(I=0, \ldots, 49)$. Now we only have to determine in an independent way the potential $\Omega(r)$ and the coefficient $C_{2}(r)$, and we have two integration constants: $K_{13}$ and $K_{24}$. The vanishing of the coefficient $C_{2}(r)$ and the constants $K_{13}$ and $K_{24}$ implies the trivial solution $\Psi_{\text {even }}=0$.

The only thing left to analyze is the integrability of $C_{2}$, or in other words, the compatibility of the equations for $C_{2}^{\prime}$ and $C_{2}^{\prime \prime}$ [Eqs. (140) and (137) respectively]. Another possibility would be to introduce Eq. (143) into Eq. (137), but in that way we would obtain an integro-differential equation for $\Omega(r)$. We follow here the first option. To that end, we compare the derivative of Eq. (140) with Eq. (137):

$$
\begin{aligned}
& \left(e_{2}+e_{1} e_{4}-e_{4}^{\prime}-e_{4}^{2}\right) C_{2} \\
& \quad+K_{24}\left(e_{1} e_{3}+\frac{f}{r^{2}}-e_{3} e_{4}-e_{3}^{\prime}\right)=0
\end{aligned}
$$

This can be written in a more convenient way as follows:

$$
\begin{aligned}
& {\left[\Omega(r)-\Omega_{*}(r)\right] C_{2}(r)+K_{24}\left\{e_{5}(r)\left[\Omega(r)-\Omega_{*}(r)\right]^{\prime}\right.} \\
& \left.\quad+e_{6}(r)\left[\Omega(r)-\Omega_{*}(r)\right]+e_{7}(r)\right\}=0
\end{aligned}
$$

where $\Omega_{*}(r)$ is given in Eq. (136) and $e_{5}(r), e_{6}(r)$, and $e_{7}(r)$ are known functions given by

$$
\begin{gathered}
e_{5}(r)=\frac{r f^{3}(r)}{\ell(\ell+1) \lambda(r)}, \\
e_{6}(r)=\frac{2 r^{2} f^{2}(r)}{\ell(\ell+1)}\left(\frac{f(r)}{\lambda(r) r}+\frac{1}{2 r}\right)^{\prime},
\end{gathered}
$$




$$
\begin{aligned}
e_{7}(r)= & \frac{f^{2}(r)}{r^{2}}+e_{5}(r)\left(\Omega_{*}(r)-\frac{\lambda^{\prime}(r)}{r}\right)^{\prime} \\
& +e_{6}(r)\left(\Omega_{*}(r)-\frac{\lambda^{\prime}(r)}{r}\right) .
\end{aligned}
$$

Equation (145) can in principle provide an expression for the coefficient $C_{2}$ [different from the one obtained in Eq. (143)]. But it is not guaranteed that such an expression would be compatible with the expressions that we have for its derivatives [Eqs. (140) and (137)]. Given that this equation is the compatibility between $C_{2}^{\prime}$ and $C_{2}^{\prime \prime}$, we just need to check the compatibility with $C_{2}^{\prime}$ [Eq. (140)]. To that end, we can take the derivative of Eq. (145) and compare with Eq. (140). Actually, if we use Eq. (140) to eliminate $C_{2}^{\prime}$ from the derivative of Eq. (145) we obtain a new relation that has the same form as Eq. (145). In principle, one can repeat this process an arbitrary number of times to get a chain of relations of the form

$$
\Pi_{n}[r, \Omega] C_{2}(r)+K_{24} \Xi_{n}[r, \Omega]=0,
$$

where $\Pi_{n}$ and $\Xi_{n}$ are coefficients that depend on the radial coordinate and on the potential function $\Omega(r)$. Using Eq. (140), it is easy to find a recurrence for these coefficients:

$$
\begin{aligned}
& \Pi_{n}[r, \Omega]=\Pi_{n-1}^{\prime}[r, \Omega]+e_{4}(r) \Pi_{n-1}[r, \Omega], \\
& \Xi_{n}[r, \Omega]=\Xi_{n-1}^{\prime}[r, \Omega]+e_{3}(r) \Pi_{n-1}[r, \Omega] .
\end{aligned}
$$

The case $n=0$ corresponds to Eq. (145) and $n=1$ to its first-order derivative after using Eq. (140) to eliminate $C_{2}^{\prime}$. Considering the $n=0$ and $n=1$ equations, there are two possibilities: (i) $C_{2}(r)=K_{24}=0$; (ii) the resultant of the system for $\left(C_{2}(r), K_{24}\right)$ vanishes, that is

$$
\Pi_{0} \Xi_{1}-\Pi_{1} \Xi_{0}=0 .
$$

Given that $\Pi_{0}=\Omega(r)-\Omega_{*}(r)$ and that [from Eq. (150)] $\Pi_{1}=\Omega^{\prime}(r)-\Omega_{*}^{\prime}(r)+e_{4}(r)\left(\Omega(r)-\Omega_{*}(r)\right)$, it is clear that $\Omega(r)=\Omega_{*}(r)$ is always a solution. In any case, Eq. (152), becomes an ODE for the potential function $\Omega(r)$. In this case, $n=0$, it is a nonlinear second-order equation for $\Omega(r)$. In principle this equation determines the form of $\Omega(r)$, which in general will be different from $\Omega_{*}(r)$ although $\Omega_{*}(r)$ is a particular solution. It turns out that the form of this ODE for $\Omega(r)$ is quite similar to the analogous equation that we have obtained in the odd-parity case [see Eq. (91)]. We find that this ODE for $\delta \Omega(r)=\Omega(r)-\Omega_{*}(r)$ is given by

$$
\begin{gathered}
K_{24}\left[\left(\hat{e}_{5}(r) \frac{\delta \Omega^{\prime}(r)}{\delta \Omega(r)}\right)^{\prime}+\left(\frac{\hat{e}_{7}(r)}{\delta \Omega(r)}\right)^{\prime}\right. \\
\left.+\hat{e}_{6}^{\prime}(r)+\hat{e}_{3}(r)-\delta \Omega(r)\right]=0,
\end{gathered}
$$

where we have introduced several definitions for the coefficients that appear in this equation. First, we have taken advantage that the coefficient $e_{4}(r)$ can be written as a total derivative [see Eq. (142)] to introduce the new coefficient $f_{4}(r)$ as follows:

$$
e_{4}(r)=\left(\ln f_{4}(r)\right)^{\prime} \Rightarrow f_{4}(r)=\frac{r f^{2}(r)}{\lambda(r)} .
$$

Then, the coefficients that appear in Eq. (153) are defined using $f_{4}(r)$ in the following way:

$$
\begin{gathered}
\hat{e}_{5}(r)=\ell(\ell+1) \frac{e_{5}(r)}{f_{4}(r)}=f(r), \\
\hat{e}_{6}(r)=\ell(\ell+1) \frac{e_{6}(r)}{f_{4}(r)}=2 \lambda(r) r\left(\frac{f(r)}{\lambda(r) r}+\frac{1}{2 r}\right)^{\prime}, \\
\hat{e}_{7}(r)=\ell(\ell+1) \frac{e_{7}(r)}{f_{4}(r)} \\
=\ell(\ell+1) \frac{\lambda(r)}{r^{3}} \\
+\hat{e}_{5}(r)\left(\Omega_{*}(r)-\frac{\lambda^{\prime}(r)}{r}\right)^{\prime} \\
+\hat{e}_{6}(r)\left(\Omega_{*}(r)-\frac{\lambda^{\prime}(r)}{r}\right), \\
\hat{e}_{3}(r)=\ell(\ell+1) \frac{\left.e_{3}(r)\right|_{\Omega=\Omega_{*}}}{f_{4}(r)} \\
=-\left[\Omega_{*}(r)+\frac{\lambda(r)-(\ell+2)(\ell-1)}{r^{2}}\right] .
\end{gathered}
$$

At this point, it is important to remark that the equation for $\delta \Omega(r)$ in the even-parity case, Eq. (153), has exactly the same structure as the corresponding equation for the oddparity case [Eq. (91)]. The only differences are the expressions for the functions of $r$ that appear in them.

If we now consider the next relation in Eqs. (150) and (151), namely $n=2$, it can be seen that by combining it with the other two $(n=0$ and $n=1)$ we obtain more ODEs for the potential function $\Omega(r)$; this time these ODEs are nonlinear third-order ones. By using the second-order ODE for $\Omega(r)$ that comes from the analysis of the cases $n=0$ and $n=1$, the (two) third-order equations for $\Omega(r)$ are identically satisfied, which ends the analysis.

As in the odd-parity case, we have to study the two branches that appear. The first branch is characterized by:

$$
\Omega(r)=\Omega_{*}(r),
$$

where $\Omega_{*}(r)$ is now given by Eq. (136). Then, Eq. (135) is automatically satisfied. Moreover, if we introduce this expression for $\Omega(r)$ into Eq. (145), we must have that 
$K_{24} e_{7}(r)=0$. Taking into account that this has to be valid for any $r$, and that in general $e_{7}(r) \neq 0$, we must have

$$
K_{24}=0 .
$$

Then, from Eq. (143), we find an expression for the coefficient $C_{2}$ :

$$
C_{2}(r)=K_{2} \frac{r f^{2}(r)}{\lambda(r)}
$$

This finishes the developments for the first branch. The potential for the even-parity perturbations in this case is given in Eq. (136). It turns out that in the case of a Schwarzschild background, this potential is the well-known Zerilli potential of Eq. (58). And in the case of a maximally symmetric background it is the centrifugal barrier potential in Eq. (95). Finally, the most general master function is

$$
\begin{aligned}
\Psi_{\text {even }}(t, r)= & K_{2}\left\{\frac{r f^{2}(r)}{\lambda(r)} h_{11}+\frac{r}{2}\left[\frac{\ell(\ell+1)}{2} G(t, r)+K(t, r)\right]\right. \\
& \left.-\frac{f(r)}{\lambda(r)}\left[\ell(\ell+1) \mathrm{J}_{1}(t, r)+r^{2} K^{\prime}(t, r)\right]\right\} \\
& +\frac{K_{13}}{\lambda(r)}\left\{r\left(\dot{K}(t, r)+\frac{\lambda(r)}{2} \dot{G}(t, r)-\frac{f^{\prime}(r)}{r} \mathrm{~J}_{0}\right)\right. \\
& \left.+f(r)\left(h_{01}(t, r)-\mathrm{J}_{0}^{\prime}(t, r)+\dot{\mathrm{J}}_{1}(t, r)\right)\right\} .
\end{aligned}
$$

Using the definitions introduced in Sec. IIE we can rewrite the master function as follows:

$\Psi_{\text {even }}(t, r)=\frac{\ell(\ell+1)}{4} K_{2} \Psi_{\mathrm{ZM}}(t, r)-K_{13} \Psi_{\mathrm{EN}}(t, r)$,

where $\Psi_{\mathrm{ZM}}(t, r)$ is the Zerilli-Moncrief master function given in Eq. (48) and $\Psi_{\mathrm{EN}}(t, r)$ is another master function (not known as far as we can say) given by

$$
\begin{aligned}
\Psi_{\mathrm{EN}}(t, r)= & \frac{r}{\lambda(r)}\left\{\frac{f(r)}{r}\left[\mathrm{~J}_{0}^{\prime}(t, r)-h_{01}(t, r)-\dot{\mathrm{J}}_{1}(t, r)\right]\right. \\
& \left.+\frac{f^{\prime}(r)}{r} \mathrm{~J}_{0}-\dot{K}(t, r)-\frac{\lambda(r)}{2} \dot{G}(t, r)\right\}
\end{aligned}
$$

which can be rewritten in a completely covariant form as follows:

$$
\Psi_{\mathrm{EN}}(t, r)=\frac{1}{\lambda(r)} t^{a}\left(r \tilde{K}_{: a}-\tilde{h}_{a b} r^{b}\right)
$$

where $\tilde{h}_{a b}$ and $\tilde{K}$ are the gauge-invariant quantities introduced in Eqs. (42) and (43) respectively. Hence, $\Psi_{\mathrm{EN}}(t, r)$ is also a gauge-invariant master function that can be written in a covariant way. By using the perturbative field equations it is possible to show that the master function $\Psi_{\mathrm{EN}}(t, r)$ turns out to be proportional to the time derivative of the Zerilli-Moncrief master function:

$$
t^{a} \Psi_{\mathrm{ZM}: a}=2 \Psi_{\mathrm{EN}} .
$$

This ends the analysis of the first branch.

In the second branch, when $\Omega(r) \neq \Omega_{*}(r)$, by virtue of Eq. (135) we must have

$$
K_{13}=0 .
$$

In this case, Eq. (145) provides a second expression for $C_{2}(r)$ that has to be compared with the other expression, Eq. (143). The outcome of this comparison is the secondorder nonlinear ODE for $\Omega(r)$ that is given in Eq. (153). Moreover, in this branch, the only nonzero coefficients of $\Psi_{\text {even }}$ are $C_{2}(r)$ [Eq. (143) with $\Omega(r)$ satisfying the nonlinear ODE of Eq. (153)], $C_{4}(r)$ [Eq. (130)], $C_{5}(r)$ [Eq. (133)], $C_{6}(r)$ [Eq. (134)], $C_{18}(r)$ [Eq. (123)], and $C_{20}$ [Eq. (131)]. Introducing these expressions into $\Psi_{\text {even }}$ we can write the result in the following form:

$$
\Psi_{\text {even }}(t, r)=\left(K_{2}+K_{24} \Sigma(r)\right) \Psi_{\mathrm{ZM}}(t, r)-\frac{K_{24}}{2} \tilde{K}(t, r),
$$

where

$\Sigma=\frac{1}{\ell(\ell+1)}\left[\frac{\lambda-(\ell+2)(\ell-1)}{2 r}-\int_{r} d r^{\prime} \Omega\left(r^{\prime}\right)\right]$,

and $\tilde{K}(t, r)$ is the gauge-invariant combination of metric perturbations introduced in Eq. (43). Therefore, the evenparity master function in this second branch is also gauge invariant. Actually, it is a linear combination of the Zerilli master function and another master function that is a combination of the Zerilli master function and $\tilde{K}(t, r)$, which depends on the potential $\Omega(r)$. The potential, in turn, satisfies the nonlinear ODE in Eq. (153). As in the oddparity case, this equation can be simplified [see Eq. (107)]. To that end, we have to write it in terms of the potential difference $\delta V=f \delta \Omega$ [see Eq. (56)], use the expressions for $\hat{e}_{3}$ and $\hat{e}_{6}$, which satisfy

$$
\hat{e}_{3}+\hat{e}_{6}^{\prime}-f^{\prime \prime}=0,
$$

and finally, we have to exchange derivatives with respect to $r$ with derivatives with respect to the tortoise coordinate. After doing all this, we arrive at a simplified equation that looks exactly like the one for the odd-parity case [see Eq. (107)]

$$
\left(\frac{\delta V_{, x}}{\delta V}\right)_{, x}+2\left(\frac{V_{, x}^{\text {even }}}{\delta V}\right)_{, x}-\delta V=0,
$$


where $V^{\text {even }}=f \Omega_{*}$ is the Zerilli potential. This ends the analysis of the even-parity case.

\section{CONCLUSIONS AND FUTURE PROSPECTS}

In this paper, we have carried out a study of all of the possible master functions and equations for the perturbations of vacuum spherically symmetric spacetimes. The only assumptions made in this study are the following: (i) The master functions are linear combinations of the metric perturbations and their first-order derivatives. (ii) The coefficients of those linear combinations only depend on the radial areal coordinate. (iii) The master functions satisfy a wave-type equation associated with the two-dimensional metric of the Lorentzian manifold tangent to the spheres of symmetry, and with a potential that is determined by the perturbative Einstein equations.

The outcome of this study produces two branches of solutions: (a) The first branch corresponds to the already known results, with the exception of the even-parity case, for which we have found a new master function independent of the Zerilli-Moncrief one, $\Psi_{\mathrm{ZM}}$, and which we have denoted by $\Psi_{\mathrm{EN}}$. For both parities, the most general master function is a linear combination (with constant coefficients) of two independent master functions. These master functions can be taken to be the Regge-Wheeler and the Cunningham-Prince-Moncrief master functions, $\left(\Psi_{\mathrm{RW}}, \Psi_{\mathrm{CPM}}\right)$, in the odd-parity case and $\left(\Psi_{\mathrm{ZM}}, \Psi_{\mathrm{EN}}\right)$ in the even-parity case. On the other hand, the potentials are the known ones: the Regge-Wheeler (odd-parity) and the Zerilli (even-parity) potentials. (b) The second branch was essentially unknown and, in contrast with the first branch, there are infinite possible potentials, different from the ones already known (first branch). The set of possible potentials corresponds to the solutions of a nonlinear differential equation which has the same form for both parities [see Eqs. (107) and (171)]. The master functions are again a linear combination (with coefficients depending only on the radial area coordinate) of two independent master functions. In the odd-parity case, they can be taken to be the Cunningham-Price-Moncrief master function and a new one that is a combination of $\Psi_{\mathrm{CPM}}$ and $\Phi_{\mathrm{ON}}$ that includes the potential function [see Eqs. (102) and (105)]. The evenparity case follows the same pattern, and the most general master function can be taken to be a linear combination of the Zerilli-Moncrief master function and another new master function made out of a combination of $\Psi_{\mathrm{ZM}}$, $\Phi_{\mathrm{EN}}$, and the potential function [see Eqs. (163) and (165)].

Apart from the construction of the master functions and equations (potentials), it is important to remark other findings that came out from our developments: (i) The flow of the argument is the same for the two parities despite the different number of variables and equations that describe the metric perturbations in the two cases. (ii) Gauge invariance: all the master functions and equations turn out to be gauge invariant, which is something that we did not impose. In this sense, it is important to remark that we have always worked on a general gauge. The emerging gauge invariance is then due to the physical/ geometric character of the (master) wave equations. This shows the important role played by the master functions, which in some sense encode the true degrees of freedom of the (perturbative) gravitational field. (iii) Despite the fact that in many places we have carried out the calculations using a specific class of coordinate systems, we have been able always to restore full covariance with respect to the $1+1$ Lorentzian metric $g_{a b}$ [see Eq. (20)]. (iv) For both parities, in the first branch, one of the independent master functions can be taken to be the time derivative of the other one [see Eqs. (100) and (166)]. (v) In the case when the background is maximally symmetric, the odd-parity and even-parity potentials of the first branch are identical. In the case of the second branch, the set of possible potentials are the same for the two parities. This means that the maximal symmetry has a strong impact on the possible set of master equations and functions. There are still two branches, but they are identical for both parities. (vi) We have always worked in the time domain. This has the advantage that at any moment we can obtain results in the frequency domain by introducing the standard substitution: $\Psi(t, r) \rightarrow e^{i \omega t} \phi(r)$.

Our analysis is quite general, in the sense that it is based on a few assumptions (see Sec. III), and complete, in the sense that it unveils the full content of the (first-order) perturbative approach to vacuum spherically symmetric spacetimes. Within our knowledge, no similar analysis has been carried out before. The systematic construction of master functions and equations we have followed can be applied directly to other different scenarios in spherical symmetry, in particular to systems involving matter fields (see [32,33] for a general approach): point particle [13], electromagnetic fields (see, e.g., [79-84]), perfect fluids $[85,86]$, etc. Within general relativity it can also be applied to spacetimes with a different number of dimensions, in particular it would be interesting to study the case of three spacetime dimensions, where we have the BañadosTeitelboim-Zanelli (BTZ) black hole [87] (which is asymptotically $\mathrm{AdS}^{3}$ ), and for which there are analytic solutions for the quasinormal frequencies and wave functions [88]. Again within general relativity, one can try to follow the procedure for second- and higher-order perturbations $[49,50,89]$ of spherically symmetric spacetimes. This is particularly interesting taking into account that the equations for higher-order perturbations usually contain the same differential operators of the background as the ones for the first-order perturbations. Finally, this procedure can also be applied to perturbations of spherically symmetric spacetimes in other theories of gravity (see, e.g., [90-94]).

Another interesting question is whether we can apply a similar procedure in the case of the Kerr metric [53] and other axially symmetric spacetimes. That would make 
contact with the Bardeen-Press master function [77] for the Schwarzschild spacetime, which is not included in our analysis since it contains second-order derivatives of the metric perturbations. In any case, it is clear that for Kerr perturbations we should allow for the presence of secondorder derivatives.

On the other hand, the results of our study lead to a number of questions. In particular, what is the meaning of the infinite set of possible master functions and equations that appear in the second branch of solutions. We clarify this question in a forthcoming paper [95], where we analyze in detail the connection between this plethora of master equations and master functions and show that all of them are related by Darboux transformations. Nevertheless, the Darboux transformation that connects them has to be interpreted in a more general context than the classical Darboux transformation which is normally introduced in the context of Sturm-Liouville problems, with self-adjoint operators. In this sense, in [95] we show the crucial role played by the equations for the potentials of the second branch, Eqs. (107) and (171).

\section{ACKNOWLEDGMENTS}

We thank José Luís Jaramillo for enlightening discussions regarding the dynamics of perturbed black holes. C. F. S. is supported by Contracts No. ESP2017-90084-P and No. PID2019-106515 GB-I00/AEI/10.13039/ 501100011033 (Spanish Ministry of Science and Innovation) and No. 2017-SGR-1469 (AGAUR, Generalitat de Catalunya). We are grateful for the COST Action CA16104 Gravitational waves, black holes and fundamental physics (GWverse) as a forum of discussion where part of this work originated and for a Short Term Scientific Mission award to M. L. that allowed us to complete this work. M. L. also acknowledges support from an Angelo della Riccia fellowship. We have used the Mathematica software [96] to implement the mathematical machinery related with Einstein's equations for spherically symmetric spacetimes shown in the appendixes. Using this Mathematica implementation we have checked most of the computations necessary for this paper.

\section{APPENDIX A: DIFFERENTIAL PROPERTIES OF SPHERICAL HARMONICS}

All the spherical harmonics (scalar, vector, and tensor) we use in this paper are determined once we prescribe the scalar spherical harmonics (see, e.g., [97,98]). The vector and tensor spherical harmonics used in this paper can then be obtained via Eqs. (25)-(28). To recover the equations and results of this paper, we only need to use some differential identities that they satisfy. The even-parity vector harmonics $Y_{A}^{\ell m}$ satisfy the following differential identities:

$$
\begin{gathered}
\Omega^{A B} Y_{A \mid B}^{\ell m}=-\ell(\ell+1) Y^{\ell m}, \\
\Omega^{B C} Y_{B \mid C A}^{\ell m}=-\ell(\ell+1) Y_{A}^{\ell m}, \\
\Omega^{B C} Y_{A \mid B C}^{\ell m}=[1-\ell(\ell+1)] Y_{A}^{\ell m} .
\end{gathered}
$$

The odd-parity vector harmonics $X_{A}^{\ell m}$ satisfy similar differential identities:

$$
\begin{gathered}
\Omega^{A B} X_{A \mid B}^{\ell m}=0, \\
\Omega^{B C} X_{A \mid B C}^{\ell m}=[1-\ell(\ell+1)] X_{A}^{\ell m}, \\
\Omega^{B C} X_{B \mid A C}^{\ell m}=X_{A}^{\ell m} .
\end{gathered}
$$

On the other hand, the (symmetric) even-parity tensor harmonics $T_{A B}^{\ell m}$ and $Y_{A B}^{\ell m}$ satisfy the following differential identities:

$$
\begin{gathered}
\Omega^{B C} T_{B C \mid A}^{\ell m}=2 Y_{A}^{\ell m}, \\
\Omega^{B C} T_{A B \mid C}^{\ell m}=Y_{A}^{\ell m}, \\
\Omega^{C D} T_{A B \mid C D}^{\ell m}=-\ell(\ell+1) T_{A B}^{\ell m}, \\
\Omega^{C D} T_{C D \mid A B}^{\ell m}=Y_{A B}^{\ell m}-\ell(\ell+1) T_{A B}^{\ell m}, \\
\Omega^{B C} Y_{B C \mid A}^{\ell m}=0, \\
\Omega^{B C} Y_{A B \mid C}^{\ell m}=-\frac{(\ell+2)(\ell-1)}{2} Y_{A}^{\ell m}, \\
\Omega^{C D} Y_{A B \mid C D}^{\ell m}=[4-\ell(\ell+1)] Y_{A B}^{\ell m} .
\end{gathered}
$$

Finally, the (symmetric) odd-parity tensor harmonics $X_{A B}^{\ell m}$ satisfy the following differential identities:

$$
\begin{gathered}
\Omega^{B C} X_{B C \mid A}^{\ell m}=0, \\
\Omega^{B C} X_{A B \mid C}^{\ell m}=-\frac{(\ell+2)(\ell-1)}{2} X_{A}^{\ell m}, \\
\Omega^{C D} X_{A B \mid C D}^{\ell m}=[4-\ell(\ell+1)] X_{A B}^{\ell m} .
\end{gathered}
$$

\section{APPENDIX B: MULTIPOLAR COMPONENTS OF GEOMETRIC PERTURBATIVE QUANTITIES}

We give expressions of the main quantities that we need to analyze the perturbative vacuum (with cosmological constant) Einstein equations (see [48] for complementary expressions). The components of the perturbation of the Christoffel symbols (these are tensors from the point of 
view of the background spacetime), introducing the spherical harmonic decomposition of the metric perturbations into Eq. (3) are

$$
\begin{aligned}
\delta \Gamma_{b c}^{a}= & \frac{1}{2} g^{a d}\left(h_{c d: b}-h_{b c: d}+h_{b d: c}\right) Y, \\
\delta \Gamma_{b A}^{a}= & \left(\frac{1}{2} p_{b}^{a}+g^{a c} \mathbf{J}_{[c: b]}-\frac{r_{b}}{r} \mathbf{J}^{a}\right) Y_{A} \\
& +\left(g^{a c} h_{[c: b]}-\frac{r_{b}}{r} h^{a}\right) X_{A},
\end{aligned}
$$

$$
\begin{aligned}
\delta \Gamma_{A B}^{a}= & \left(r r^{b} p_{b}^{a}-\frac{\ell(\ell+1)}{2} \mathrm{~J}^{a}-\frac{1}{2}\left(r^{2} K\right)^{: a}\right) T_{A B} \\
& +\left(\mathrm{J}^{a}-\frac{1}{2}\left(r^{2} G\right)^{: a}\right) Y_{A B}+\left(h^{a}-\frac{1}{2} h_{2}: a\right) X_{A B},
\end{aligned}
$$

$$
\delta \Gamma_{a b}^{A}=\frac{1}{r^{2}}\left(\mathrm{~J}_{a: b}-\frac{1}{2} p_{a b}\right) Y^{A}+\frac{1}{r^{2}} h_{a: b} X^{A},
$$

$$
\begin{gathered}
\delta \Gamma_{B a}^{A}=\frac{1}{2 r^{2}}\left(\left(r^{2} K\right)_{: a}-2 r r_{a} K\right) T_{B}^{A}+\frac{1}{2 r^{2}}\left(\left(r^{2} G\right)_{: a}-2 r r_{a} G\right) Y_{B}^{A}+\frac{1}{2 r^{2}}\left(h_{2: a}-2 \frac{r_{a}}{r} h_{2}\right) X_{B}^{A}+\frac{\ell(\ell+1)}{2 r^{2}} h_{a} Y \epsilon^{A}{ }_{B} . \\
\delta \Gamma_{B C}^{A}= \\
\frac{1}{2} K\left(\Omega_{B}^{A}{ }_{B} Y_{C}+\Omega^{A}{ }_{C} Y_{B}-\Omega_{B C} Y^{A}\right)+\frac{1}{2} G \Omega^{A D}\left(Y_{C D \mid B}-Y_{B C \mid D}+Y_{B D \mid C}\right) \\
+\frac{h_{2}}{2 r^{2}} \Omega^{A D}\left(X_{C D \mid B}-X_{B C \mid D}+X_{B D \mid C}\right)+\frac{r^{a}}{r}\left(\mathrm{~J}_{a} Y^{A}+h_{a} X^{A}\right) \Omega_{B C} .
\end{gathered}
$$

Now, in the same way that we have the harmonic decomposition of the perturbed Christoffel symbols in terms of the metric perturbation harmonics, we can write the harmonic decomposition of the Riemann tensor in terms of the harmonic decomposition of the perturbations of the Christoffel symbols. Then, from Eq. (4) we obtain

$$
\begin{aligned}
& \delta R^{a}{ }_{b c d}=\delta \Gamma^{a}{ }_{b d: c}-\delta \Gamma_{b c: d}^{a}, \\
& \delta R^{a}{ }_{b c A}=\delta \Gamma^{a}{ }_{b A: c}-\delta \Gamma^{a}{ }_{b c \mid A}+r r^{a} \Omega_{A B} \delta \Gamma^{B}{ }_{b c}+\frac{r_{b}}{r} \delta \Gamma^{a}{ }_{A c}, \\
& \delta R^{a}{ }_{b A B}=\delta \Gamma_{b B \mid A}^{a}-\delta \Gamma^{a}{ }_{b B \mid A}-r r^{a}\left(\Omega_{A C} \delta \Gamma_{b B}^{C}-\Omega_{B C} \delta \Gamma^{C}{ }_{b A}\right), \\
& \delta R^{a}{ }_{A b c}=\delta \Gamma^{a}{ }_{c A: b}-\delta \Gamma^{a}{ }_{b A: c}-\frac{r_{b}}{r} \delta \Gamma^{a}{ }_{c A}+\frac{r_{c}}{r} \delta \Gamma^{a}{ }_{b A}, \\
& \delta R^{a}{ }_{A b B}=\delta \Gamma^{a}{ }_{A B: b}-\delta \Gamma^{a}{ }_{b A \mid B}-\frac{r_{b}}{r} \delta \Gamma^{a}{ }_{A B}+r r^{a} \Omega_{B C} \delta \Gamma^{C}{ }_{A b}-r r^{c} \delta \Gamma^{a}{ }_{b c} \Omega_{A B}, \\
& \delta R^{a}{ }_{A B C}=\delta \Gamma^{a}{ }_{A C \mid B}-\delta \Gamma^{a}{ }_{A B \mid C}-r r^{a}\left(\Omega_{B D} \delta \Gamma^{D}{ }_{A C}-\Omega_{C D} \delta \Gamma^{D}{ }_{A B}\right)+r r^{b}\left(\Omega_{A B} \delta \Gamma_{b C}^{a}-\Omega_{A C} \delta \Gamma_{b B}^{a}\right), \\
& \delta R^{A}{ }_{a b c}=\delta \Gamma_{a c: b}^{A}-\delta \Gamma_{a b: c}^{A}+\frac{r_{b}}{r} \delta \Gamma_{a c}^{A}-\frac{r_{c}}{r} \delta \Gamma_{a b}^{A}, \\
& \delta R^{A}{ }_{a b B}=\delta \Gamma^{A}{ }_{a B: b}-\delta \Gamma^{A}{ }_{a b \mid B}+\frac{r_{a}}{r} \delta \Gamma_{B b}^{A}+\frac{r_{b}}{r} \delta \Gamma_{B a}^{A}-\frac{r_{c}}{r} \delta \Gamma^{c}{ }_{a b} \delta_{B}^{A}, \\
& \delta R^{A}{ }_{a B C}=\delta \Gamma^{A}{ }_{a C \mid B}-\delta \Gamma^{A}{ }_{a B \mid C}+\frac{r_{b}}{r}\left(\delta \Gamma^{b}{ }_{a C} \delta^{A}{ }_{B}-\delta \Gamma^{b}{ }_{a B} \delta^{A}{ }_{C}\right), \\
& \delta R^{A}{ }_{B a b}=\delta \Gamma_{B b: a}^{A}-\delta \Gamma_{B a: b}^{A}, \\
& \delta R_{B a C}^{A}=\delta \Gamma_{B C: a}^{A}-\delta \Gamma_{B a \mid C}^{A}-\frac{r_{b}}{r} \delta \Gamma_{a B}^{b} \delta_{C}^{A}-r r^{b} \delta \Gamma^{A}{ }_{a b} \Omega_{B C}, \\
& \delta R^{A}{ }_{B C D}=\delta \Gamma_{B D \mid C}^{A}-\delta \Gamma^{A}{ }_{B C \mid D}+\frac{r_{a}}{r}\left(\delta \Gamma_{B D}^{a} \delta_{C}^{A}-\delta \Gamma_{B C}^{a} \delta_{D}^{A}\right)+r r^{a}\left(\Omega_{B C} \delta \Gamma^{A}{ }_{a D}-\Omega_{B D} \delta \Gamma^{A}{ }_{a C}\right) .
\end{aligned}
$$


From here we can find the harmonic decomposition of the perturbations of the Ricci tensor by introducing the perturbations of the Christoffel symbols in terms of the metric perturbation harmonics [Eqs (B1)-(B6)]. The result is

$$
\begin{aligned}
\delta R_{a b}= & {\left[h_{c(a: b)}: c-\frac{1}{2} h_{a b: c}: c-\frac{1}{2} h_{: a b}+\frac{r^{c}}{r}\left(h_{a c: b}+h_{b c: a}-h_{a b: c}\right)-K_{: a b}-\frac{2}{r} r_{(a} K_{: b)}+\frac{\ell(\ell+1)}{2 r^{2}}\left(h_{a b}-2 \mathrm{~J}_{a: b}\right)\right] Y, } \\
\delta R_{a A}= & {\left[\frac{1}{2} h_{a b}: b-\frac{1}{2} h_{: a}+\frac{r_{a}}{2 r} h+\frac{1}{2} \mathrm{~J}^{c}: c a-\frac{1}{2} \mathrm{~J}_{a: c}{ }^{c}-\frac{r_{a}}{r} \mathrm{~J}^{b}: b+\frac{r^{b}}{r} \mathrm{~J}_{b: a}+\left(\frac{{ }^{2} R}{4} g_{a b}-\frac{r_{: a b}}{r}-\frac{r_{a} r_{b}}{r^{2}}\right) \mathrm{J}^{b}-\frac{1}{2} K_{: a}\right.} \\
& \left.-\frac{(\ell+2)(\ell-1)}{4} G_{: a}\right] Y_{A}+\left[\frac{1}{2} h^{c}: c a\right. \\
2 & \frac{1}{2} h_{a: c}{ }^{c}-\frac{r_{a}}{r} h^{b}: b+\frac{r^{b}}{r} h_{b: a}+\left(\frac{{ }^{2} R}{4} g_{a b}-\frac{r_{: a b}}{r}-\frac{r_{a} r_{b}}{r^{2}}\right) h^{b}+\frac{\ell(\ell+1)}{2 r^{2}} h_{a} \\
& \left.-\frac{(\ell+2)(\ell-1)}{4 r^{2}}\left(h_{2: a}-\frac{2 r_{a}}{r} h_{2}\right)\right] X_{A}, \\
\delta R_{A B}= & {\left[r r^{c} h^{a}{ }_{c: a}-\frac{r}{2} r^{a} h_{: a}+\left(r^{a} r^{b}+r r^{: a b}\right) h_{a b}+\frac{\ell(\ell+1)}{4} h-\frac{\ell(\ell+1)}{2}\left(\mathrm{~J}^{a}: a+2 \frac{r^{a}}{r} \mathrm{~J}_{a}\right)-\frac{1}{2}\left(r^{2} K\right): a: a\right.} \\
& \left.+\frac{\ell(\ell+1)}{2} K+\frac{(\ell+2)(\ell+1) \ell(\ell-1)}{4} G\right] T_{A B}+\left[\mathrm{J}^{a}: a-\frac{1}{2} r^{2} G_{: a}: a-r r^{a} G_{: a}-\left(r r^{: a}: a+r^{a} r_{a}-1\right) G-\frac{h}{2}\right] Y_{A B} \\
& +\left[h^{a}: a-\frac{1}{2} h_{2: a}: a+\frac{r^{a}}{r} h_{2: a}+\frac{\left(1-2 r^{a} r_{a}\right)}{r^{2}} h_{2}\right] X_{A B} .
\end{aligned}
$$

[1] J. M. Bardeen, Gauge-invariant cosmological perturbations, Phys. Rev. D 22, 1882 (1980).

[2] H. Kodama and M. Sasaki, Cosmological perturbation theory, Prog. Theor. Phys. Suppl. 78, 1 (1984).

[3] G. F. R. Ellis and M. Bruni, Covariant and gauge-invariant approach to cosmological density fluctuations, Phys. Rev. D 40, 1804 (1989).

[4] W. Hu and N. Sugiyama, Toward understanding CMB anisotropies and their implications, Phys. Rev. D 51, 2599 (1995).

[5] H.-P. Nollert, Quasinormal modes: The characteristic 'sound' of black holes and neutron stars, Classical Quant. Grav. 16, R159 (1999).

[6] K. D. Kokkotas and B. G. Schmidt, Quasinormal modes of stars and black holes, Living Rev. Relativity 2, 2 (1999).

[7] N. Andersson and K. D. Kokkotas, The r-mode instability in rotating neutron stars, Int. J. Mod. Phys. D 10, 381 (2001).

[8] K. D. Kokkotas and B. F. Schutz, W-modes: A new family of normal modes of pulsating relativistic stars, Mon. Not. R. Astron. Soc. 255, 119 (1992).

[9] P. C. Peters, Gravitational radiation and the motion of two point masses, Phys. Rev. 136, B1224 (1964).

[10] C. W. Misner, K. Thorne, and J. A. Wheeler, Gravitation (W. H. Freeman \& Co., San Francisco, 1973).

[11] L. Blanchet, Gravitational radiation from post-Newtonian sources and inspiralling compact binaries, Living Rev. Relativity 17, 2 (2014).
[12] A. Le Tiec, The overlap of numerical relativity, perturbation theory and post-Newtonian theory in the binary black hole problem, Int. J. Mod. Phys. D 23, 1430022 (2014).

[13] M. Davis, R. Ruffini, W. Press, and R. Price, Gravitational Radiation from a Particle Falling Radially into a Schwarzschild Black Hole, Phys. Rev. Lett. 27, 1466 (1971).

[14] O. Dreyer, B. J. Kelly, B. Krishnan, L. S. Finn, D. Garrison, and R. Lopez-Aleman, Black hole spectroscopy: Testing general relativity through gravitational wave observations, Classical Quant. Grav. 21, 787 (2004).

[15] M. Sasaki and H. Tagoshi, Analytic black hole perturbation approach to gravitational radiation, Living Rev. Relativity $\mathbf{6}$, 6 (2003).

[16] V. Ferrari and L. Gualtieri, Quasi-normal modes and gravitational wave astronomy, Gen. Relativ. Gravit. 40, 945 (2008).

[17] B. Sathyaprakash and B. Schutz, Physics, astrophysics and cosmology with gravitational waves, Living Rev. Relativity 12, 2 (2009).

[18] E. Berti, K. Yagi, H. Yang, and N. Yunes, Extreme gravity tests with gravitational eaves from compact binary coalescences: (II) Ringdown, Gen. Relativ. Gravit. 50, 49 (2018).

[19] V. Cardoso, L. Gualtieri, C. Herdeiro, U. Sperhake, P. M. Chesler et al., NR/HEP: Roadmap for the future, Classical Quant. Grav. 29, 244001 (2012).

[20] R. Brito, V. Cardoso, and P. Pani, Superradiance: New Frontiers in Black Hole Physics (Springer, New York, 2015), Vol. 906. 
[21] L. Barack et al., Black holes, gravitational waves and fundamental physics: A roadmap, Classical Quant. Grav. 36, 143001 (2019).

[22] E. Poisson, A. Pound, and I. Vega, The motion of point particles in curved spacetime, Living Rev. Relativity 14, 7 (2011).

[23] L. Barack, Gravitational self-force in extreme mass-ratio inspirals, Classical Quant. Grav. 26, 213001 (2009).

[24] L. Barack and A. Pound, Self-force and radiation reaction in general relativity, Rep. Prog. Phys. 82, 016904 (2019).

[25] K. Schwarzschild, On the gravitational field of a mass point according to Einstein's theory, Sitzungsber. Preuss. Akad. Wiss. Berlin (Math. Phys.) 7, 189 (1916), https://archive .org/details/sitzungsberichte1916deutsch/page/188/.

[26] T. Regge and J. A. Wheeler, Stability of a Schwarzschild singularity, Phys. Rev. 108, 1063 (1957).

[27] C. Vishveshwara, Stability of the Schwarzschild metric, Phys. Rev. D 1, 2870 (1970).

[28] C. Vishveshwara, Scattering of gravitational radiation by a Schwarzschild black-hole, Nature (London) 227, 936 (1970).

[29] F. J. Zerilli, Effective Potential for Even Parity ReggeWheeler Gravitational Perturbation Equations, Phys. Rev. Lett. 24, 737 (1970).

[30] F. J. Zerilli, Gravitational field of a particle falling in a Schwarzschild geometry analyzed in tensor harmonics, Phys. Rev. D 2, 2141 (1970).

[31] V. Moncrief, Gravitational perturbations of spherically symmetric systems. I. The exterior problem, Ann. Phys. (N.Y.) 88, 323 (1974).

[32] U. H. Gerlach and U. K. Sengupta, Gauge invariant perturbations on most general spherically symmetric space-times, Phys. Rev. D 19, 2268 (1979).

[33] U. H. Gerlach and U. K. Sengupta, Gauge invariant coupled gravitational, acoustical, and electromagnetic modes on most general spherical space-times, Phys. Rev. D 22, 1300 (1980).

[34] O. Sarbach and M. Tiglio, Gauge invariant perturbations of Schwarzschild black holes in horizon-penetrating coordinates, Phys. Rev. D 64, 084016 (2001).

[35] C. A. Clarkson and R. K. Barrett, Covariant perturbations of Schwarzschild black holes, Classical Quant. Grav. 20, 3855 (2003).

[36] S. Mukohyama, Gauge invariant gravitational perturbations of maximally symmetric space-times, Phys. Rev. D 62, 084015 (2000).

[37] H. Kodama and A. Ishibashi, A master equation for gravitational perturbations of maximally symmetric black holes in higher dimensions, Prog. Theor. Phys. 110, 701 (2003).

[38] V. Ferrari and B. Mashhoon, New approach to the quasinormal modes of a black hole, Phys. Rev. D 30, 295 (1984).

[39] E. Berti, V. Cardoso, and A. O. Starinets, Quasinormal modes of black holes and black branes, Classical Quant. Grav. 26, 163001 (2009).

[40] R. Konoplya and A. Zhidenko, Quasinormal modes of black holes: From astrophysics to string theory, Rev. Mod. Phys. 83, 793 (2011).

[41] J. Natario and R. Schiappa, On the classification of asymptotic quasinormal frequencies for d-dimensional black holes and quantum gravity, Adv. Theor. Math. Phys. 8, 1001 (2004).

[42] B. S. Kay and R. M. Wald, Linear stability of Schwarzschild under perturbations which are nonvanishing on the bifurcation two sphere, Classical Quant. Grav. 4, 893 (1987).

[43] B. S. Kay and R. M. Wald, Theorems on the uniqueness and thermal properties of stationary, nonsingular, quasi-free states on space-times with a bifurcate Killing horizon, Phys. Rep. 207, 49 (1991).

[44] F. Mellor and I. Moss, Stability of black holes in de Sitter space, Phys. Rev. D 41, 403 (1990).

[45] S. Chandrasekhar, The Mathematical Theory of Black Holes (Oxford University Press, New York, 1992).

[46] Y. Mino, M. Sasaki, M. Shibata, H. Tagoshi, and T. Tanaka, Black hole perturbation: Chapter 1, Prog. Theor. Phys. Suppl. 128, 1 (1997).

[47] A. Nagar and L. Rezzolla, Gauge-invariant nonspherical metric perturbations of Schwarzschild black-hole spacetimes, Classical Quant. Grav. 22, R167 (2005); Erratum, Classical Quant. Grav. 23, 4297 (2006).

[48] K. Martel and E. Poisson, Gravitational perturbations of the Schwarzschild spacetime: A practical covariant and gaugeinvariant formalism, Phys. Rev. D 71, 104003 (2005).

[49] R. J. Gleiser, C. O. Nicasio, R. H. Price, and J. Pullin, Second order perturbations of a Schwarzschild black hole, Classical Quant. Grav. 13, L117 (1996).

[50] R. J. Gleiser, C. O. Nicasio, R. H. Price, and J. Pullin, Gravitational radiation from Schwarzschild black holes: The second order perturbation formalism, Phys. Rep. 325, 41 (2000).

[51] A. Garat and R. H. Price, Gauge invariant formalism for second order perturbations of Schwarzschild spacetimes, Phys. Rev. D 61, 044006 (2000).

[52] S. A. Teukolsky, Rotating Black Holes-Separable Wave Equations for Gravitational and Electromagnetic Perturbations, Phys. Rev. Lett. 29, 1114 (1972).

[53] S. A. Teukolsky, Perturbations of a rotating black hole. 1. Fundamental equations for gravitational electromagnetic and neutrino field perturbations, Astrophys. J. 185, 635 (1973).

[54] A. Ishibashi and H. Kodama, Stability of higher dimensional Schwarzschild black holes, Prog. Theor. Phys. 110, 901 (2003).

[55] M. Dafermos, G. Holzegel, and I. Rodnianski, The linear stability of the Schwarzschild solution to gravitational perturbations, Acta Math. 222, 1 (2019).

[56] C. T. Cunningham, R. H. Price, and V. Moncrief, Radiation from collapsing relativistic stars. I. Linearized odd-parity radiation, Astrophys. J. 224, 643 (1978).

[57] C. T. Cunningham, R. H. Price, and V. Moncrief, Radiation from collapsing relativistic stars. II. Linearized even parity radiation, Astrophys. J. 230, 870 (1979).

[58] C. T. Cunningham, R. H. Price, and V. Moncrief, Radiation from collapsing relativistic stars. III. Second order perturbations of collapse with rotation, Astrophys. J. 236, 674 (1980).

[59] J. M. Stewart and M. Walker, Perturbations of spacetimes in general relativity, Proc. R. Soc. A 341, 49 (1974).

[60] R. Wald, General Relativity (University of Chicago Press, Chicago, 1984). 
[61] M. Bruni, S. Matarrese, S. Mollerach, and S. Sonego, Perturbations of space-time: Gauge transformations and gauge invariance at second order and beyond, Classical Quant. Grav. 14, 2585 (1997).

[62] C. F. Sopuerta, M. Bruni, and L. Gualtieri, Nonlinear Nparameter space-time perturbations: Gauge transformations, Phys. Rev. D 70, 064002 (2004).

[63] G. D. Birkhoff, Relativity and Modern Physics (Harvard University Press, Cambridge, MA, 1923).

[64] J. T. Jebsen, Über die allgemeinen kugelsymmetrischen Lösungen der Einsteinschen Gravitationsgleichungen im Vakuum, Ark. Mat. Ast. Fys.(Stockholm) 15, 1 (1921).

[65] J. Jebsen, On the general spherically symmetric solutions of Einstein's gravitational equations in vacuo, Gen. Relativ. Gravit. 37, 2253 (2005).

[66] S. Deser and J. Franklin, Schwarzschild and Birkhoff a la Weyl, Am. J. Phys. 73, 261 (2005).

[67] N. Voje Johansen and F. Ravndal, On the discovery of Birkhoff's theorem, Gen. Relativ. Gravit. 38, 537 (2006).

[68] J. Eiesland, The group of motions of an Einstein space, Trans. Am. Math. Soc. 27, 213 (1925).

[69] K. Schleich and D. M. Witt, A simple proof of Birkhoff's theorem for cosmological constant, J. Math. Phys. (N.Y.) 51, 112502 (2010).

[70] F. Kottler, Über die physikalischen Grundlagen der Einsteinschen Gravitationstheorie, Ann. Phys. (Germany) 56, 401462 (1918).

[71] H. Nariai, On some static solutions of Einstein's gravitational field equations in a spherically symmetric case, Sci. Rep. Tohoku Univ. Series I 34, 160 (1950).

[72] H. Nariai, On a new cosmological solution of Einstein's field equations of gravitation, Gen. Relativ. Gravit. 31, 963 (1999).

[73] J. Podolsky, The Structure of the extreme Schwarzschild-de Sitter space-time, Gen. Relativ. Gravit. 31, 1703 (1999).

[74] J. Droste, The field of a single centre in Einstein's theory of gravitation, and the motion of a particle in that field, K. Ned. Akad. Wet. Proc. Ser. B Phys. Sci. 19, 197 (1917).

[75] S. Jhingan and T. Tanaka, Improvement on the metric reconstruction scheme in Regge-Wheeler-Zerilli formalism, Phys. Rev. D 67, 104018 (2003).

[76] C. O. Lousto and R. H. Price, Head-on collisions of black holes: The particle limit, Phys. Rev. D 55, 2124 (1997).

[77] J. M. Bardeen and W. H. Press, Radiation fields in the Schwarzschild background, J. Math. Phys. (N.Y.) 14, 7 (1973).

[78] E. Newman and R. Penrose, An approach to gravitational radiation by a method of spin coefficients, J. Math. Phys. (N.Y.) 3, 566 (1962).

[79] V. Moncrief, Odd-parity stability of a Reissner-Nordstrom black hole, Phys. Rev. D 9, 2707 (1974).

[80] V. Moncrief, Stability of Reissner-Nordstrom black holes, Phys. Rev. D 10, 1057 (1974).

[81] V. Moncrief, Gauge-invariant perturbations of ReissnerNordstrom black holes, Phys. Rev. D 12, 1526 (1975).
[82] S. Chandrasekhar, On the equations governing the perturbations of the Reissner-Nordström black hole, Proc. R. Soc. A 365, 453 (1979).

[83] S. Chandrasekhar and B. C. Xanthopoulos, On the metric perturbations of the Reissner-Nordström black hole, Proc. R. Soc. A 367, 1 (1979), https://www.jstor.org/stable/79860.

[84] B. C. Xanthopoulos, Metric and electromagnetic perturbations of the Reissner-Nordström black hole, Proc. R. Soc. A 378, 73 (1981), https://www.jstor.org/stable/2397164.

[85] C. Gundlach and J. M. Martín-García, Gauge-invariant and coordinate-independent perturbations of stellar collapse. I. The interior, Phys. Rev. D 61, 084024 (2000).

[86] J. M. Martín-García and C. Gundlach, Gauge-invariant and coordinate-independent perturbations of stellar collapse. II. Matching to the exterior, Phys. Rev. D 64, 024012 (2001).

[87] M. Bañados, C. Teitelboim, and J. Zanelli, The Black Hole in Three-Dimensional Space-Time, Phys. Rev. Lett. 69, 1849 (1992).

[88] I. Ichinose and Y. Satoh, Entropies of scalar fields on threedimensional black holes, Nucl. Phys. B447, 340 (1995).

[89] D. Brizuela, J. M. Martin-Garcia, and G. A. M. Marugan, Second- and higher-order perturbations of a spherical spacetime, Phys. Rev. D 74, 044039 (2006).

[90] T. Kobayashi, H. Motohashi, and T. Suyama, Black hole perturbation in the most general scalar-tensor theory with second-order field equations. I. The odd-parity sector, Phys. Rev. D 85, 084025 (2012); Erratum, Phys. Rev. D 96, 109903 (2017).

[91] T. Kobayashi, H. Motohashi, and T. Suyama, Black hole perturbation in the most general scalar-tensor theory with second-order field equations. II. The even-parity sector, Phys. Rev. D 89, 084042 (2014).

[92] S. Bhattacharyya and S. Shankaranarayanan, Quasinormal modes as a distinguisher between general relativity and $f(R)$ gravity, Phys. Rev. D 96, 064044 (2017).

[93] S. Bhattacharyya and S. Shankaranarayanan, Quasinormal modes as a distinguisher between general relativity and $\mathrm{f}(\mathrm{R})$ gravity: Charged black-holes, Eur. Phys. J. C 78, 737 (2018).

[94] S. Bhattacharyya and S. Shankaranarayanan, Distinguishing general relativity from Chern-Simons gravity using gravitational wave polarizations, Phys. Rev. D 100, 024022 (2019).

[95] M. Lenzi and C. F. Sopuerta, Darboux covariance: A hidden symmetry of perturbed Schwarzschild black holes, arXiv:2109.00503.

[96] Wolfram Research, Mathematica 12 (2020), https://www .wolfram.com/mathematica/.

[97] M. Abramowitz and I. A. Stegun, Handbook of Mathematical Functions with Formulas, Graphs, and Mathematical Tables (Dover, New York, 1972).

[98] W. H. Press, B. P. Flannery, S. A. Teukolsky, and W. T. Vetterling, Numerical Recipes: The Art of Scientific Computing (Cambridge University Press, Cambridge, England, 1992). 